Ceumplinents of the

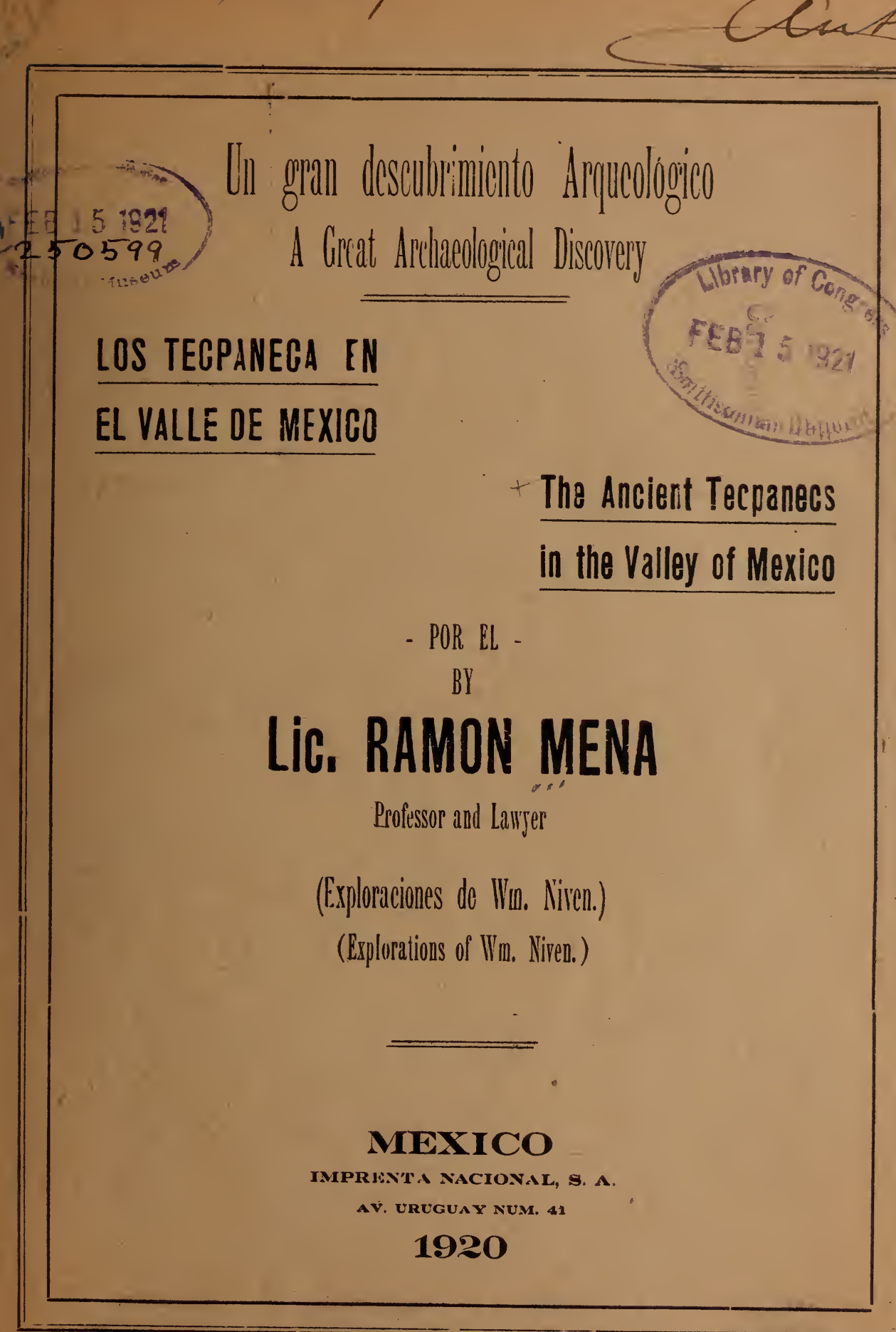

Libary of Anithomian Trothution 



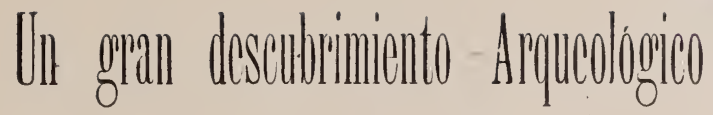 \\ A Gireat Amtheodogital Discovery}

\section{LOS TEGPANEGA EN}

\section{EL VALLE DE MEX:CO}

\section{The Ancient Tecpanecs \\ in the Valley of Mexico}

- POR El -

BI

\section{Lic, RAMON MENA}

Profissor and lanter

(I:xp'oraciones de NIm. Niren.)

(Explorations of Wm. Niven.)

MEXICO

IMPRENTA NACIONAL, S. A

AV. URUGUAY NUM 41

\section{0}




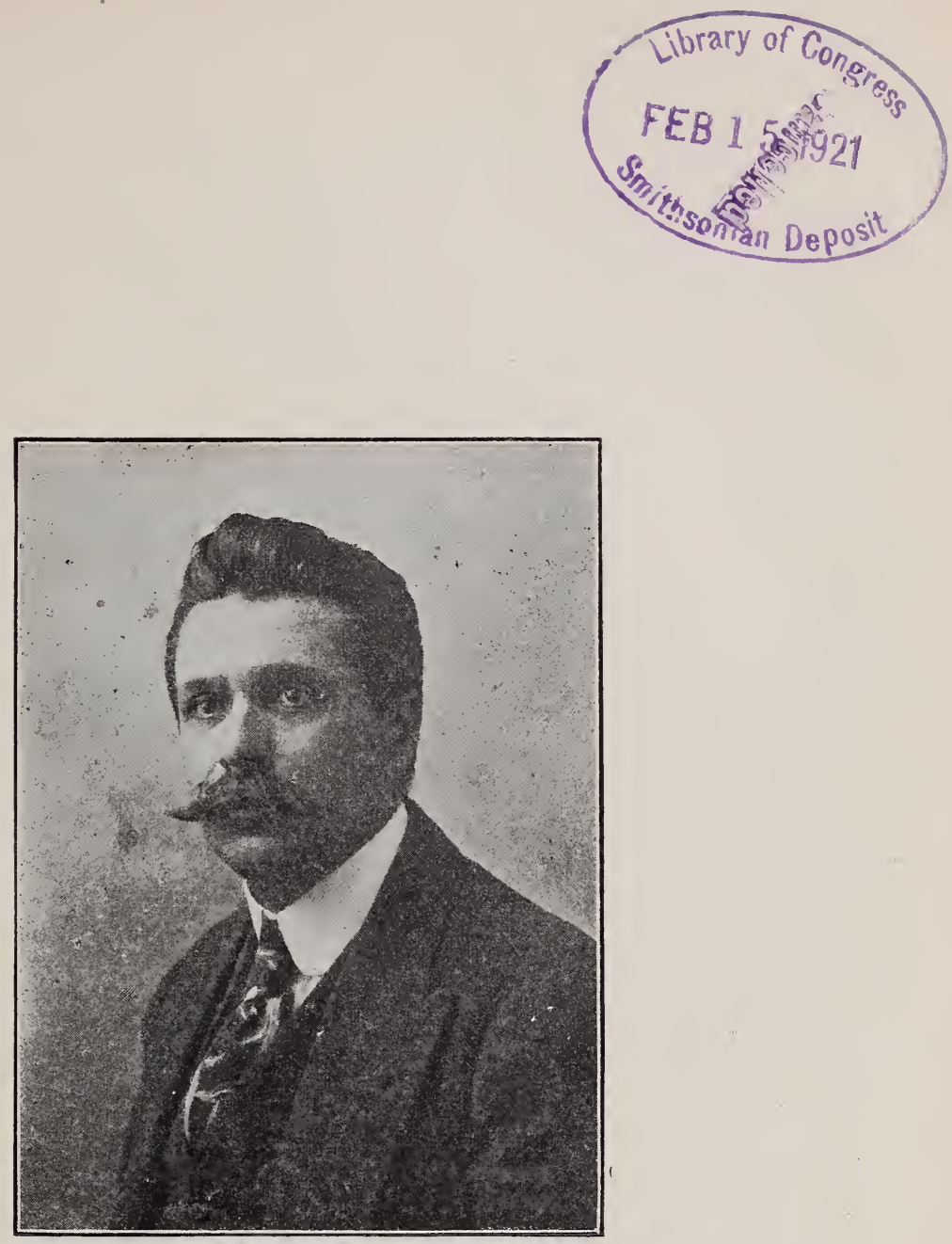

Lic. Ramón Mena.

Autor. 



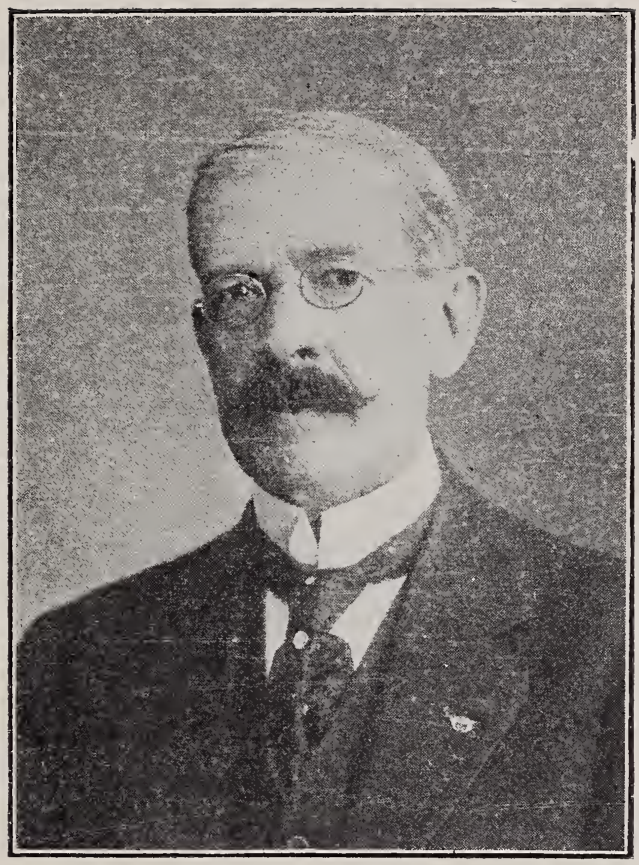

Wim. Niven.

Explorador. 



\section{LOS TEGPANEGAS EN EL VALLE DE MEXIGO}

Las exploraciones arqueológicas llavadas a término por el Profesor William Niven, durante el año 1918-1919 en una zona de la región Noroeste del Valle de México, han sido fecundas en descubrimientos de ejemplares que han arrojado no poca luz en la densa obscuridad que envuelve la actividad social de nuestros antecesores.

Metódicas y constantes las exploraciones de referencia, han tocado lugares de comprensión absolutamente TECPANECA, tales como San Juan TLILHUACA, AZCAPOTZALCO, Santiago AHUIZOCTLA, San Miguel AMANTLA, Tacuba (TLACOPAN), San Juanico, POPOTLA y San Joaquín (CACALCO).

La comprensión tecpaneca en el Valle, abarcaba, en sentir constante y uniforme de Historiadores primitivos y de Pictógrafos de Códices: Azcapozalco, Tacuba, porción de Tacubaya, Coyoacán, Magdalena y Culhuacán; pues bien, aquella uniformidad de autores, recibe comprobación plena del material arqueológico encontrado, y por tanto, queda fijada ya definitivamente, la Geografía tecpaneca en el Valle de México. El Croquis que se acompaña, ilustra.suficientemente acerca del particular, y define la relación entre tecpanecas y pedregalenses.

Cuanto a la exploración propiamente dicha, se hizo siempre, siguiendo el método estratigráfico, en el que, la experiencia de algunos años, ha fijado al Sr. Niven, en el Valle de México, los horizontes arqueológicos respectivos, con más precisión que a la Escuela Internacional de Arqueología, en 1911-12.

Véase nuestro esquema estratigráfico. Anotamos en él por primera vez, el "horizonte tecpaneca," indiscutiblemente anterior al "teotihuasano."

La importancia del horizonte tecpaneca es tal, que eslabona la éposa pedregalense, primitiva o arcaica, con la teotihuacana, y nuevamente, nos resulta el dato del Historiador, sostenido por el descubrimiento arqueológico; con efecto, el florecimiento teotihuacano, da principio con la dedicación de las célebres pirámides, en el siglo VII de J. C. y en ese siglo, largo tiempo hacía, sin duda siglos, que los tecpanecas radicaban en el Valle de México y hasta Capital tenían en Azcapotzalco; por otra parte, añadiendo a este dato que espigamos en la Crónica de las Crónicas, en 
la Historia de Orozco y Berra, añadiendo, repito, el de que, cuando Torquemada escribía su "Nonarquía Indiana," hacia 1595, o antes, los tecpaneca afirmaban estar radicados en Azcapotzalco, hacia 1565 años, resultan ya con Capital, desde el año 30 de J. C. y como quiera que para organizarse una tribu hasta llegar a constituir forma de Gobierno y tener ciudad cabeza, transcurren no solamente treinta años, sino tal vez un siglo, tendremos que los tecpaneca existen en el Valle de México, con anterioridad a J. C. Todo ello recibe confirmación, del material arqueológico encontrado y al que se contrae la presente Monografía.

\section{¿QUIENES ERAN LOS TECPANECA?}

El Códice precortesiano de la Peregrinación Azteca, nos dice que eran una de las tribus de la gran familia nahua que estuvo en Chicomoztoc y peregrinó rumbo a este Valle; la misma autoridad, nos da el nombre hieróglifo, fonético-figurativo: un tecpatl (pedernal), con lo que, dedse luego, corregimos definitivamente la gráfica de la palabra, así: TECPANECA; pues no ha faltado quien escriba tepaneca, gente de palacios, alterando la verdad.

Tecpatl, es pedernal, y necatl, es terminación gentilicia de los nombres de pueblo que acaban en pa o en tl; de manera que tecpanecatl, es la gente del pedernal, o bien, la gente de Tecpatl (lugar, rito). Según el Códice y los Historiadores, salieron de Chicomoztoc, los tecpaneca en tercer lugar, y llegaron al Valle, mucho tiempo antes que los azteca; pero al peregrinar, se dividieron en grupos, por lo que, aun en su Reino, aparecen llegando en diferentes épocas: primeramente y con mayor antigüedad, los de Atzcapotzalco, después, los de Tlacopan. Como las tribus o parcialidades nahuatlacas, traían sus Deidades, aquella denominada Tlacatecpanecatl, creemos_era la de los tecpaneca, y el material arqueológico a estudio, comprueba el que al establecerse la parcialidad, ya tenía una religión y prácticas rituales, y sin duda conocimientos cosmogónicos, por eso vemos a las diversas tribus nahuas, celebrar sobre la marcha, la fiesta cíclica en varias ocasiones, y a los tolteca, de la misma familia etnográfica y linguiística, hacer la corrección del calendario, 133 años antes de J. C.

Cuanto al tipo tecpaneca, lo presentaremos al concluir el estudio del material arqueológico, como resultante necesaria de aquel.-Veamos:

\section{MATERIAL ARQUEOLOGICO.}

De julio de 1918 a fines del año de 1919, los repetidos hallazgos de objetos arqueológicos, por el Sr. Niven, amigo y colega en estudios llamaron fuertemente mi atención, por el estilo especial, antes no conocido, por la manufactura y por la profundidad de los hallazgos y los yacimientos en los que aparecían los objetos. Fueron los primeros, una plancha ovalar, sin pintura ni bruñido ni color y que llevaba en la parte media del reverso, un vástago que hace ángulo con la cara misma y permite a la plancha mantenerse a manera de atril. Describiendo en su oportunidad la plancha, dijimos referirse a ritos de Xochiquetzal y Chicomexochitl, presentando la cuerda florida (xochimecatl); el manjar 
u ofrenda florida ritual y diversas deidades de los días "FLOR." Hablamos entonces de la actitud de las figurillas de cuerpo entero, colocadas lateralmente, que se compaginan con la fiesta cíclica, y advertimos la perfección en el modelado y dibujo de los tocados, contrastando con

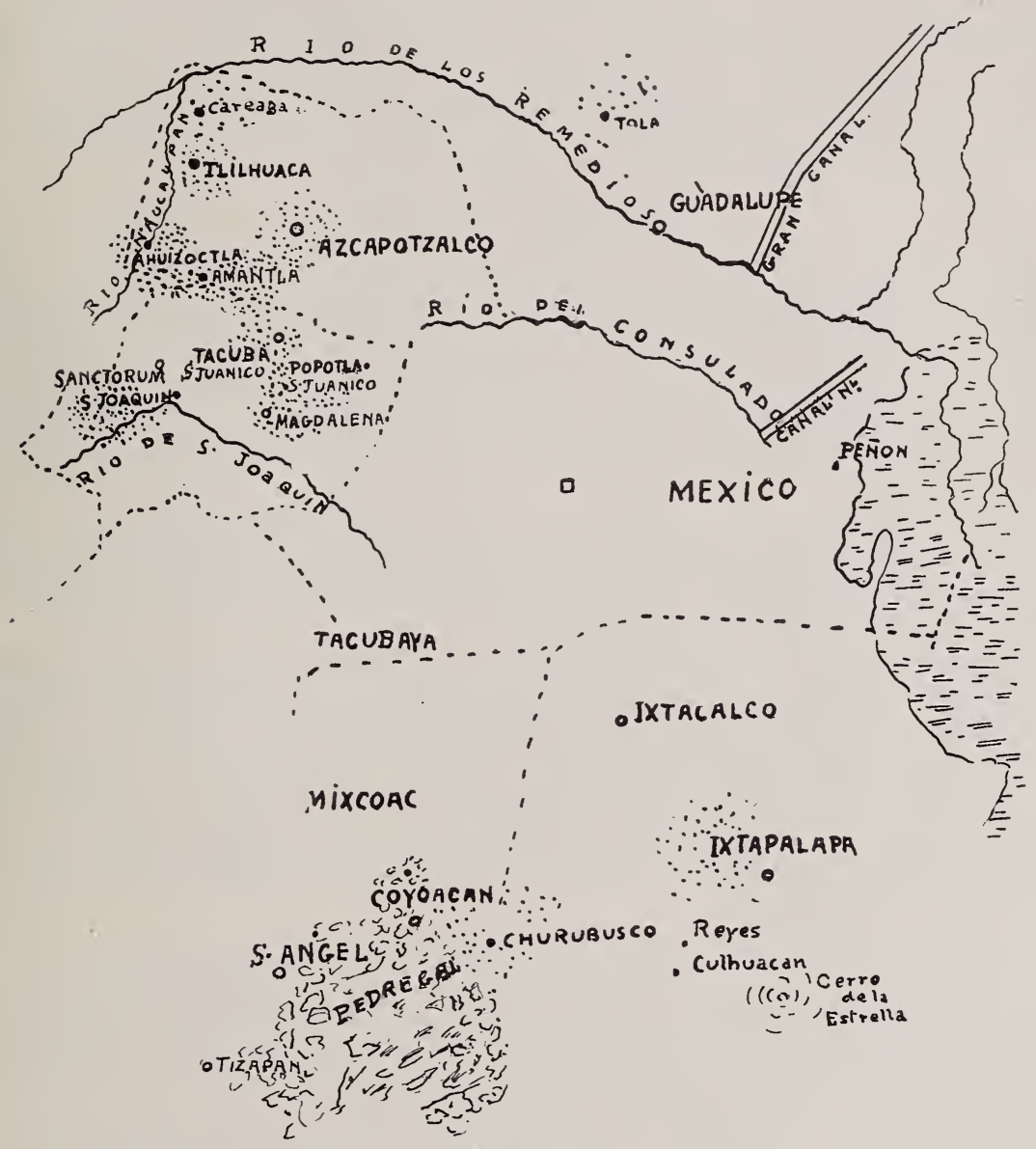

(Fig. 1.)

las extremidades de las figuras humanas y la factura de las flores $y$ de la cuerda, todo ello imperfecto, casi primitivo; precisamos ser los tipos antropológicos, tecpanecas, y cuanto a la estratigrafía, dijimos, haber sido encontrada la valiosa pieza, a poco más de tres metros de profundidad, en un yacimiento de arena, con cenizas y restos de adobe y tepetate de muros caídos o de pisos de casas o templos. Mide la plancha $0 . \mathrm{m} 22$ por $0 \mathrm{~m} .15$. Su cocción es incompleta, y la pasta fina y bien batida. El horizonte estratigráfico de hallazgo es el tecpaneca, en un segundo período. La técnica general es la de pastillaje: Cuerda, flores, vasos y fi- 
guras antropomórficas han sido modeladas aisladamente y sobrepuestas en crudo... más caso sui géneris: las cabecitas y los cuerpos, han sido hechos en molde. (Figura 3.) La procedencia, es Santiago Ahuizoctla, perteneciente a Azcapotzalco.

De la misma procedencia y yacimiento y profundidad son los vasos de las Figuras 4 y 5 ; el 4 es del tipo apaztli; tiene de alto 16 centímetros, y de diámetro 24: está adornada con la cuerda de flores, dejando campo en el vientre para figurillas de buhos y de deidades de los días FLOR, colocadas por pastillaje. Aparecen cabezas de deidad con anteojera, que son representaciones arcaicas de Xochipilli, Dios de las Flores y del Fuego. El tlacatecolotl ostenta el vientre ornado de flores.

La figura 5, es un vaso del tipo comitl, esto, es, olla de igual procedencia, yacimietno y profundidad que los ejemplares descriptos. La manufactura desigual, sin torno; la decoración es del xochimecatl, y sobre el vientre, una mascarilla de molde, rodeada de flores de estilos diferentes: el primitivo y el que en el caso llamaremos azteca, flores que iacen un total de 7 , dando la palabra Chicomexochitl, dios masculino de la Xochiquetzal. 13 centímetros tiene de alto este vaso, y 7 de diámetro. Fn este y el anterior, en el yacimiento, se encontró además de lo expuesto, grava.

Del Chicomexochitl, no podemos pasar inadvertido el tipo racial, cara larga, oval y frente alta, plana y hendida al centro en la línea del cabello. En fin, una cabecita absolutamente tecpaneca, de un período de los 3 que integran esta interesante cultura.

La Figura 6, es un grupo de madres, llevando sus hijos: la manera de cargarlos, es todo un documento etnográfico; la señalada con la letra A, a, arriba y centro de la Fig., lleva mellizos: uno a brazos, el otro, a la espalda. La madre está desnuda y muestra los senos; la cabeza lleva tocado de bandas de tela, entre las que asoma el cabello y rematan lateralmente en orejeras discoidales. Ancha o gruesa banda sale de la porción posterior del tocado y se liga a la cintura, dejando hueco para colocar uno de los mellizos.

El rostro de la madre tiene expresión: los párpados bajos hacia el niño, y la boca sonriente, dentro del tipo mongoloide racial.

Como acontece en esta cultura, las extremidades son desdibujadas y de mal modelado, lo que no ocurre en las cabezas; sin embargo, no acontece lo mismo en la técnica de niños.

En B, abajo, no se trata de mujer viviente, snio de una Diosa reconocible en las flores que ostenta en brazos y pies, y en no llevar a brazos niño vivo, sino un penate.

En C, abajo, la figurilla tiene la máscara de Xochipilli, nariguera de una flor esferoidal y grandes orepas discoidales y tocado de una flor. (¿Es Xochiquetzal?) De cualquier modo, es de etapa anterior a las A y B.

Sorprende el que los tocados, las orejeras y la faja ventral de los ninos, hayan perdurado hasta los azteca del tiempo del Conquistador Cortés.

La A, mide casi 6 centímetros de alto, por 6 de ancho de brazo a brazo, y 11 de longitud. 
La B, mide 6 cents. de alto y 7 de nacho, con longitud de 5, pues sus piernas están recojidas.

La C, tiene 5 cents. de alto por 5 y medio de ancho y 7 y medio de longitud.

En todas, la pasta es fina, amarillenta y de cocción incompleta.

Todas proceden de San Juan Tlilhuaca, en la línea divisoria entre Azcapotzalco y el Estado de México. Encontradas a 4 y 4 metros 50 centímetros de profundidad, en un yacimiento de grava, arena y cenizas: la arena y la grava menuda rellenaban los huecos de piernas y brazos, y la arena muy adherida a los huecos y salientes de la cara.

\section{RITOS SECRETOS.}

La Figura 7 enseña un grupo de estas figurillas humanas de horizonte estratigráfico tecpaneca, encontradas indistintamente en Tlilhuaca, San Juanico, Amantla y Ahuizoctla, así como en Tepalcatitla, lugar situado entre Amantla y Ahuizoctla, y en todos esos lugares, en un yacimiento siempre con grava y arena. A las exploraciones, concurrí con el descubridor Sr. Niven, y puedo testificar la autenticidad de los yacimientos, intocados antes de ahora, así como de los hallazgos que hoy enriquecen el americanismo.

Los caracteres generales de las figurillas son como los de las antes descriptas; las flores abundan en tocados y collares, y es notable, el que las figurillas, masculinas y femeninas, bien de Deidades, bien humanas, tengan siempre una mano cubriendo los labios, y la otra sobre un muslo. Otras veces, la mano es sobre el pecho. Y esta señal todavía existente en ritos secretos modernos, pero que derivan de remotísimos tiempos, no es raro haya existido entre los tecpaneca, en el solemne y complicado rito de los Dioses de las Flores y del Fuego. Sorprende encontrar en este horizonte a Huehueteotl, el Dios viejo, el más antiguo del Panteón americano, el que lleva la cara surcada de arrugas, indicando su vejez; pues la figurilla de este Dios, aparece haciendo la seña secreta...!!

Y qué decir de la Xochiquetzal, qué desde los primeros períodos tecpanecas, aparece ya con su quetzal en el tocado y sus gargantillas de flores? En nuestras figuras, el quetzal no ha conservado el pico, está roturado; el resto del tocado, es indiferentemente de Xochiquetzal y de Xochipilli, pero fino, dibujado, estilizado ya! en cambio los collares y las flores, son primitivos, y el cuerpo está substituído por un cono toscamente modelado y con aplicaciones por pastillaje, de cuerdas y de flores, y la Diosa, también presenta el ademán secreto. Y no es todo: hay figurillas arcaicas, del primer período tecpaneca, del que sigue inmediatamente al pedregalense, con el que estuvo, tal vez, en contacto (hace diez mil años) y que ostenta la actitud ritual a que vinimos contrayéndonos; y las figurillas humanas, masculinas y femeninas de estos ritos secretos, llevan al descubierto los órganos genitales como si el rito estuviera relacionado con el culto fálico, del que hablaremos adelante. 


\section{EL CULTO AL FALO.}

El grupo recogido en la Figura 8, presenta un tocado cónico con ornatos florales. De tiempo atrás sabemos que los tocados cónicos hacen referencia al culto fálico; por lo menos entre los azteca y entre los totonacos, aun cuando no eran los únicos en rendir culto al falo; puesto que entre los maya-quiché y los tarascos, está bien definido y documentado. Y ahora, encontramos la existencia de semejante culto entre los tecpaneca de los tiempos más atrazados; con efecto, buena parte de las figuras humanas de tocado cónico, tienen al descubireto el pene, en erección, y hasta la figura femenina que también lleva tocado cónico, hace ostentación de sus partes pudendas; pero hay más: la pieza.. está como desprendiéndose de un objeto, que para la percepción vulgar, es una sonaja, pero que parece ser una gota o escurrimiento seminal; con efecto, en el relieve de Jonuta (Núm 20 de la Galería de Monolitos del Museo $N$. de Arqueología) los falos que asoman a guisa de clavillos en la cabeza del Saceraote, eyaculan evidentemente y la producción seminal es en gotas ovoideas, de forma semejante a esta pieza.

Dada la importancia del producto en la generación, es congruente el encerrar o figurar en ella, la deidad de que es atributo.

La relación entre el falo y la generación las eslabonaron, y supervivieron en el Panteón azteca, en la Coatlicue, por ejemplo, en la Vía Lactea: Iztacmixcoatl, de donde el culto y las representaciones de las culebras entre los aztecas, obedeciendo todo ello a un origen fálico, ni más ni menos y que se inicia entre los tecpaneca, según lo demuestran los ejemplares examinados. Son de idénticos caracteres generales que los de figuras antecedentes, y sus dimensiones oscilan entre 6 y 9 centimetros.

Han sido encontrados en Tlilhuaca, en el yacimiento descripto y con la arena muy adherida.

\section{OTRAS FIGURAS HUMANAS.}

Dos tipos de varones y uno de mujer ofrecemos en la Figura 9; de los primeros, el A, es notable por el tocado, cuanto por tener un aditamento que lo hace sostenerse en atril; el cabello cotrado en fleco sobre la frente, deja dos mechones que caen a lo largo de la oreja, dejando libre la oreja discoidal; bandas de petate envuelven la cabeza; de la cara, la nariz está desgastada, por virtud de agentes exteriores; lleva collar de tres flores, mide 14 cents. de alto.

La cara está vaciada en molde.

El B, es hecho en dos moldes diversos: uno para la cabeza y mano derecha, y otro para el resto del cuerpo. La expresión de la faz, por su hieratismo y tipo tecpaneca, es sorprendente; cojinetes de flores alternan con el cabello que cae a la izquierda. El busto desnudo, y brazos y piernas con fundas de flores. Banda de flores terciada al pecho, y riquísimo maxtlatl de fleco almenado; además, banda a la cintura, con el elegante nudo típico de las deidades del Fuego, cuyo rostro tiene. Mide la figurilla 15 cents. por 7 de ancho; es plana en la cara posterior. 
Arrchaeological Stratigraphy of Valley of México.

\begin{tabular}{|c|c|c|c|c|}
\hline $\begin{array}{l}\text { Depth } \\
\text { in meters. }\end{array}$ & $\begin{array}{l}\text { Thickness } \\
\text { of the } \\
\text { strata }\end{array}$ & Beds. & Cultures. & Objects found. \\
\hline 1.95 & 0.40 & $\begin{array}{l}2 \text { Regular } \\
\text { of } 0.85 \text { each. }\end{array}$ & Aztec only. & $\begin{array}{l}\text { Caps of cement.-Offering vase.- } \\
\text { Incense burners. }\end{array}$ \\
\hline 2.50 & 0.55 & 1 irregular. & Teotihuareán. & $\begin{array}{l}\text { Painted layers.-Human figures ar- } \\
\text { ticulated.-Fragments of colored } \\
\text { pottery.-Vases of seratched decor- } \\
\text { ation.-Offering vase. }\end{array}$ \\
\hline 4.50 & 0.66 & $\begin{array}{l}3 \text { irregular } \\
\text { Pockets, } \\
\text { Gravel and } \\
\text { sand. }\end{array}$ & Tecpanec. & $\begin{array}{l}\text { Vases imperfectly modeled by the } \\
\text { fingers, and primitive flowers.-Hu- } \\
\text { man figure heads well modeled, pri- } \\
\text { mitive bodies adhered clay. }\end{array}$ \\
\hline 5.50 & 100 & $\begin{array}{l}1 \text { regular; } \\
\text { volcanie } \\
\text { ashes. }\end{array}$ & Primitive. & $\begin{array}{l}\text { Vases animal forms.-Decoration } \\
\text { made by fingers.-Deformed human } \\
\text { figures, oblique eyes hollow, and } \\
\text { made by adhered clay. }\end{array}$ \\
\hline 7.00 & 1.00 & $\begin{array}{l}\text { Geological } \\
\text { layer. }\end{array}$ & & \\
\hline
\end{tabular}

\section{Estratigrafia Arqueológica del Valle de México.}

\begin{tabular}{|c|c|c|c|c|}
\hline $\begin{array}{l}\text { Profundidad } \\
\text { en metros. }\end{array}$ & $\begin{array}{l}\text { Espesor } \\
\text { de los } \\
\text { estratos }\end{array}$ & $\begin{array}{l}\text { Yacimien- } \\
\text { tos. }\end{array}$ & $\begin{array}{c}\text { Culturas } \\
\text { sncontradas. }\end{array}$ & Ejemplares. \\
\hline 1.95 & 0.40 & $\begin{array}{l}2 \text { regulares } \\
\text { de } 0.85 \text {. }\end{array}$ & Azteca. & $\begin{array}{l}\text { Pisos de hormigón; porta-ofrendas, } \\
\text { incensarios. }\end{array}$ \\
\hline 2.50 & 0.55 & 1 irregular. & $\begin{array}{l}\text { Teotihua- } \\
\text { cana. }\end{array}$ & $\begin{array}{l}\text { Pisos pintados, figurillas humanas } \\
\text { articuladas, tiestería policroma; va- } \\
\text { sos de decoración raspada.-Porta- } \\
\text { ofrendas. }\end{array}$ \\
\hline 4.50 & 0.66 & $\begin{array}{l}3 \text { irregulares. } \\
\text { Bolsones. } \\
\text { Grava y arena }\end{array}$ & Tecpaneca. & $\begin{array}{l}\text { Vasos mal modelados, decoración un- } \\
\text { gulada y floral primitiva.-Figuri- } \\
\text { llas humanas de cabeza bien mode- } \\
\text { lada y cuerpo primitivo; predomi- } \\
\text { na el pastillaje. }\end{array}$ \\
\hline 5.50 & 1.00 & $\begin{array}{l}1 \text { regular } \\
\text { cenizas } \\
\text { volcánicas. }\end{array}$ & Primitiva. & $\begin{array}{l}\text { Vasos zoomórficos; decoración ungu- } \\
\text { lada.-Figurillas humanas defor- } \\
\text { mes, ojos oblícuos, huecos y por } \\
\text { pastillaje. }\end{array}$ \\
\hline 7.00 & 1.00 & $\begin{array}{l}\text { Piso } \\
\text { Geológico. }\end{array}$ & - & \\
\hline
\end{tabular}


Con C, marcamos una figura mujeril con altura de 11 centímetros y 7 de ancho. El rostro se acerca en tipo y técnica de modelado, a lo pedregalense; el tocado, casi oriental, es característico tecpaneca, y de la ropa, el huipilli (camisa) y el cueitl (enagua) tienen un carácter eminentemente búdhico. Este estilo de vestir, pero adornadas las prendas con cintas, bandas, dibujos, subsisitió hasta los azteca, y lo encontramos entre los teotihuacanos, que como llevamos demostrado, son muy posteriores a los tecpaneca.

No obstante el período remoto a que pertenece la figurilla, lleva collar de tres flores, y bajo, dos gargantillas de 5 cuentas cada una: cuatro pequeñas, dos en cada extremidad, y una larga al centro: el número 5 (macuilli) puede referirse a Macuilxochitl.

Cabe advertir que ninguna de estas tres figurillas es una deidad en sí. Son personas con los atavíos de las deidades, y esta mujeril, fue sin duda penate, porque en la porción baja de la cara posterior, muestra un pequeño pivote que ayuda a sostenerla en la estación vertical.

Hay otras figurillas, generalmente de penates: su cabeza bien modelada con el tocado que le corresponde y el cuerpo, invariablemente sin brazos ni piernas y acercándose a la forma de una pirámide truncada cuya base mayor está hacia arriba; estos cuerpos, bien lisos, bien con líneas señalando el sitio de inserción de brazos y muslos, y remotamente, llevan en la porción anterior grecas esgrafiadas. Son de un solo molde.

\section{LOS MOLDES}

Los moldes de cabezas, cuerpos, penates, pintaderas, y aún cuando raros, de figurillas de cuerpo entero, son gruesos, bien cocidos, y es indudable, que están tomados con arcilla fina bien pulverizada y batida, y en fresco, sobre un ejemplar modelado cuidadosamente. El molde fue una necesidad impuesta por el culto, de ahí la cantidad grande de penates y de cabezas de deidades. Hubo también, el retrato familiar, por lo menos, el tipo fue copiado por el escultor tecpaneca en su medio así solamente se explica, el que las deidades, tengan un marcado tipo racial, no obstante los tocados, orejeras y collares, que alteran la fisonomía.

De los moldes, hacían vaciados, y comprimían fuertemente la pasta, de arcilla plástica, que conocían bien, y dejaban secar al sol, dentro del molde, con lo que, al perder el agua la pasta, se desprendía el vaciado, que aún oreaban a la sombra, para someterlo a la coacción, bien directamente sobre leños encendidos, bien dentro de hornos, aun cuando no conocemos todavía el proceso; el fuego no era directo en el segundo caso, esto sí lo sabemos porque en el horizonte tecpaneca hemos encontrado unas piecesillas a manera de fondo de vaso con tres pies; el objeto pasó a los azteca, y aun en este siglo XX, lo utilizan los alfareros indígenas, para colocar sus piezas cerámicas dentro del horno, en forma que el fuego no las ataque directamente, aún se les denomina.... ...en lengua azteca o mexicana, y vulgarmente les nombran en español, caballitos.

Los tecpaneca no hicieron relieves en moldes, para aplicarlos por 


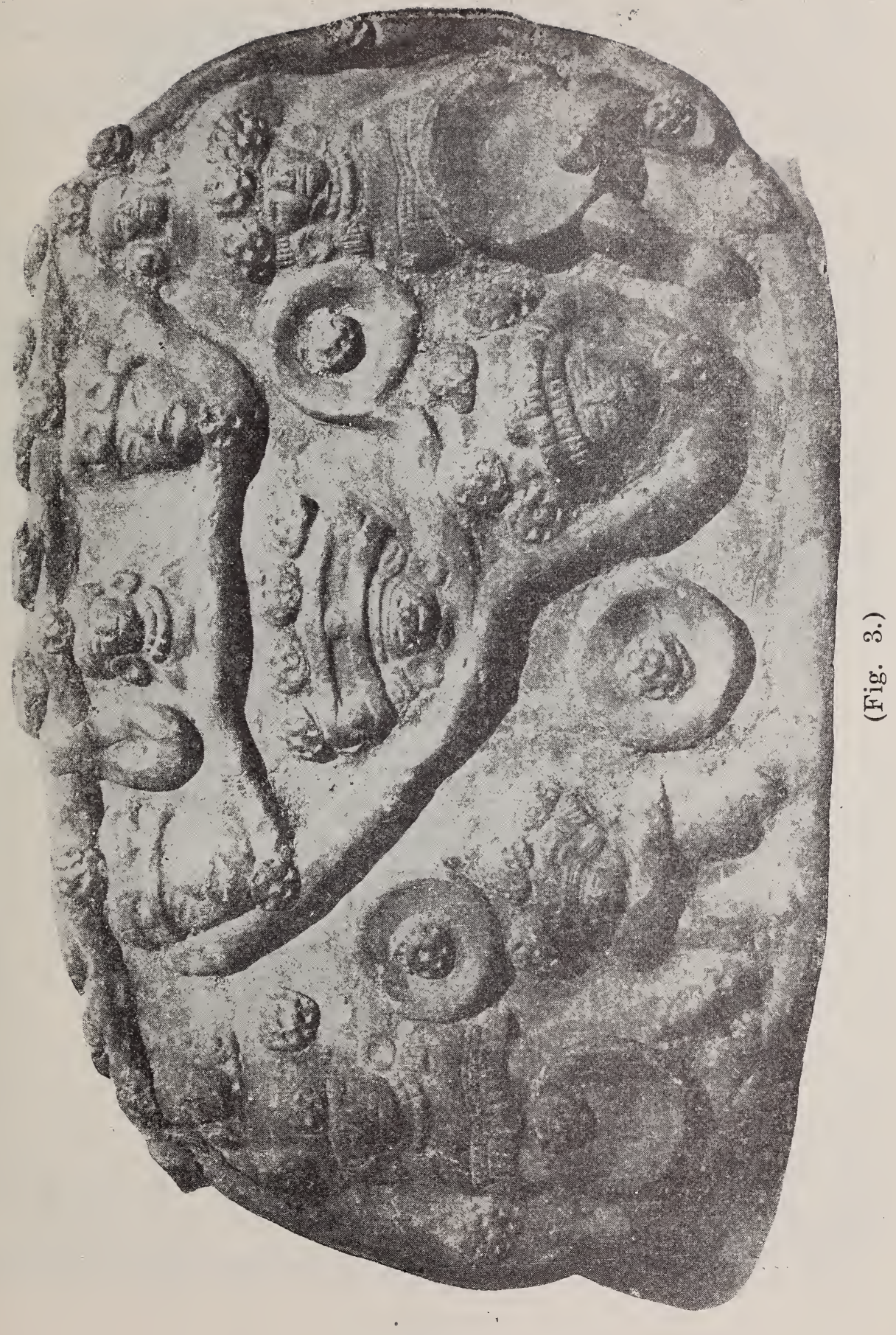


pastillaje sobre vasos o incensarios; por lo menos esta técnica teotihuacana $\mathrm{y}$ azteca, no ha aparecido en cuanto llevamos explorado del horizonte estratigráfico citado.

El molde de las pintaderas no es sino de la porción hueca que ha de dar el vaciado, así es que el mamelón para servirse de la pieza, es aplicado por pastillaje, antes de la cocción.

\section{¿HECHICEROS O ANIMALES MITOLOGICOS?}

Damos una curiosa Figura 11, con sujetos humanos de dos moldes, llevando cabeza, probablemente de mono, con un tocado extraño, en tal forma, que invirtiendo la figura, por la disposición del tocado y de los ojos, resulta la cara como si no se hubiera invertido. Hay una, en la que el cuerpo, que en la posición normal, aparece sentado, al invertirlo, resulta acrobático y la cabeza en la disposición citada, y éste, es de lo más primitivo.

Cabezas del mismo tipo humanizadas, por decir así, las encontramos en cuerpos de cuadrúpedos, y alguna hasta con la cresta de Macuilxochitl. ¿Qué es esto? Los ejemplares, son auténticos, de los yacimientos y procedencias descriptos desde el principio de la presente Monografía.

Visto que de tan antiguo como $\in \mathrm{s}$ el tiempo de los tecpaneca, existían ritos y cosmogonías interesantes y multiplicados, no va fuera de lugar, creer sean los hechiceros o nigrománticos, que cambiaban forma de cuerpo y se transformaban frecuentemente. De cualquier modo, el interés de estas piezas, resulta grande.

\section{A S O S.}

Todo, en la cultura analizada es revelador: los vasos no podían faltar en ésta, y los de la primera etapa exclusivamente destinados al culto de las Deidades florales, están adornados de fiores, en el bocal y en el vientre. Cuanto a morfología, la forma en "fondo de saco," mal modelada, sin reborde y con aplicaciones de flores, pasa a la olla (comitl), de líneas y proporciones bien encontradas, pero de modelado rudimental y con sus flores primitivas, aplicadas por pastillaje. Hay la tendencia a reproducir la forma humana y la de algunos animales en estos vasos, principalmente en los "fondo de saco;" así tenemos las de buho y de cara de un hombre, todo por pastillaje sobre el vientre de los vasos. Cabe advertir que dichos vasos, frecuentemente son pequeños, como juguetes, o acaso, modelos.

En otro período, encontramos la forma xicalli, con grueso pastillaje, decorativo del vientre; también la misma forma, con pies en asa, 3 pies, y aun 4, los que no pocas veces alternan así: dos en asa, y dos en mamelón. Esta forma de pies no es nahua. sino tarasca, y tal vez son remembranzas del paso de los nahuas por Michuacan. Hasta la decoración, esgrafiada y a mano libre y de líneas en zig-zag o de circulillos radiados, recuerdan lo primitivo y la técnica ingenua de las primeras épocas. (Megalitos de El Pedregal).

De los vasos expuestos en la Figura 12, tienen una decoración ventral 


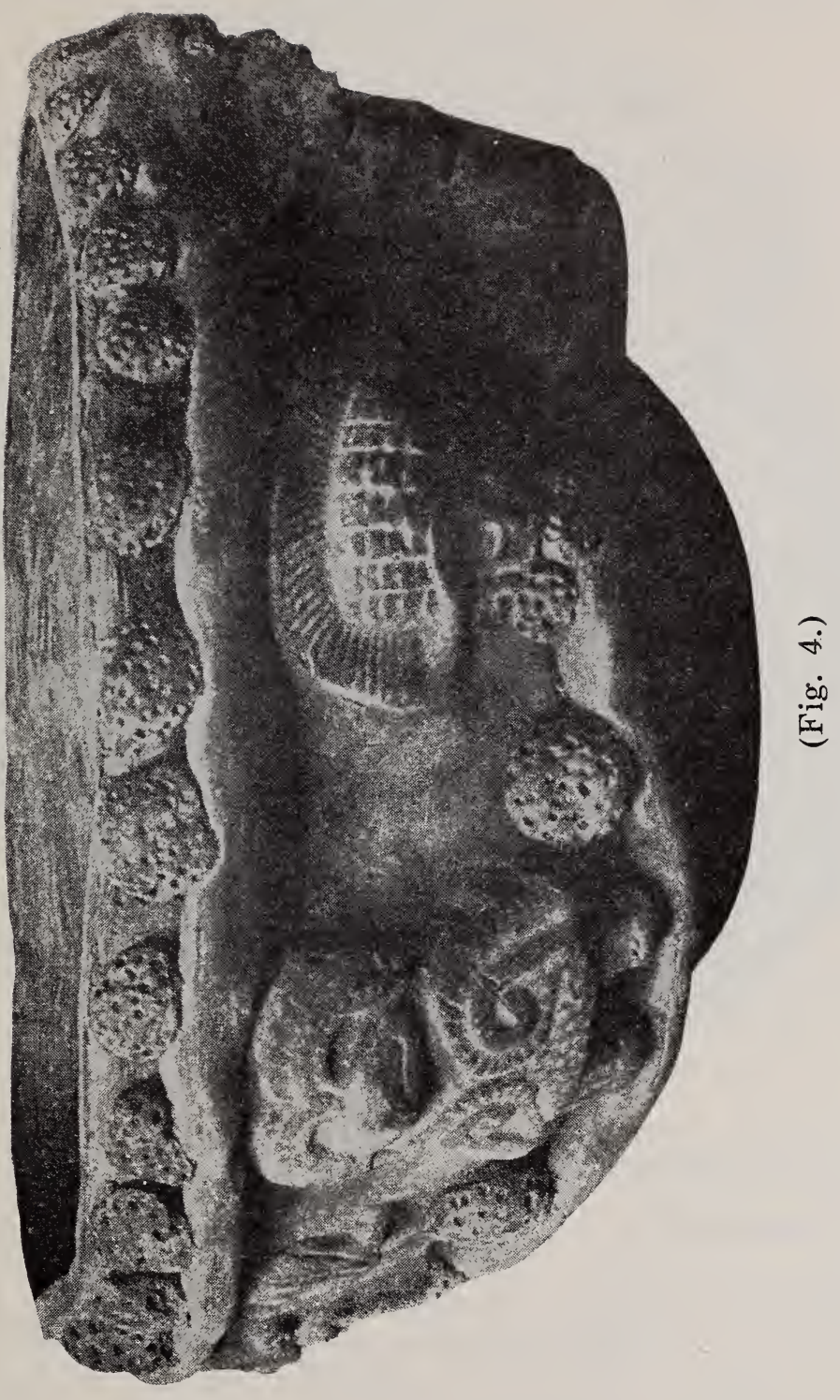

en hueco, misma que subsistió en la época testihuacana, y que resulta equilibrada y elegante por su misma sobriedad.

Comparada este Figura, con las 4 y 5 , apreciaremos la evolución morfológica del vaso entre los tecpaneca. El color, no lo hemos encontrado sobre ningún vaso de esta cultura, pero en las figurillas huma- 
nas, representaciones del Dios del Fuego, sí aparecen los colores amarillo y rojo, alternados en banaas.

\section{EVOLUCION DEL TIPO TECPANECA.}

En el horizonte tecpaneca, principia a ser definido un tipo antropológico sui géneris, que sin embargo, ofrece una remembraza pedregalense; a medida que se asciende en el horizonte citado, las facciones, por virtud de un mejoramiento en la técnica del modelado y en el arte del retrato, adquieren verdad y finura; pero la cabeza, que no requiere sino la observación, fija su silueta en 3 períodos: frente chata hendida en el vertex. Frente chata alta, y frente abombada.

La primera, corresponde a la mayor profundidad, al yacimiento que eslabona con el pedregalense.

La segunda y la tercera, se perpetúan, conviven con los teotihuacanos, a quienes alcanzan en sus mismos dominios, y las encontramos todavía entre los azteca del siglo XVI cortesiano. Así queda explicada la presencia del tipo tecpaneca en Teotihuacán, endonde la gran cantidad de CABECITAS ahí existentes, presentan el característico tipo antes citado. Con lo que tenemos, que la extensión cultural teotihuacana, no llegó a los tecpaneca, quienes llevamos establecido, fueron anteriores, y en su horizonte, ni tipo antropológico ni cerámica doméstica ni ritual teotihuacanos aparecen.

No es este el lugar de hacer un estudio comparativo entre el tipo antropológico de las 7 tribus nahuatlacas; más podemos decir a priori, que presentarían matices de distinción, engendrados por hábitos, alimentación y medio, indudables.

Del tipo tecpaneca podemos afirmar, que desde su primera etapa, cuando el escultor aplicaba por imperfecto pastillaje ojos, nariz, labios, y ornatos, puede definirse la característica antropológica de la cabeza.

La tendencia al prognatismo, existe en el tipo tecpaneca, pero como tendencia; que un prognatismo definido y determinante, no lo encontramos, ni en la primera etapa tecpaneca, no, las cabecitas son ortógnatas.

La depresión frontal fue artificial? Así lo parece, aun cuando no tenemos material cranaeano del tipo, a fin de comprobar aserto semejante.

\section{GEOGRAFIA Y ESTRATIGRAFIA REGIONALES.}

La Geografía tecpaneca, está fijada en el Croquis de la Figura 1 y ahi puede seguirse la evolución tecpaneca de Sur Este a Noroeste, dentro del Valle, y comprendiendo en su estancia y paso por el Sur, las relaciones de que hemos hablado con los pedregalenses, y así avanzaron bajando de las estribaciones del Ajusco a las lomas de Mixcoac, de Tarubaya y San Joaquín; y finalmente a la planicie de Azcapotzalco, Capital del Reino que empujó a los aztecas al. Pedregal y luego a las aguas del Lago, endonde habrían de fundar la gran Tenochtitlán.

En la época tecpaneca, la hidrogafía de su región no era la que ahora conocemos; desde luego, las aguas del Lago de. Tezcoco, llegaban 


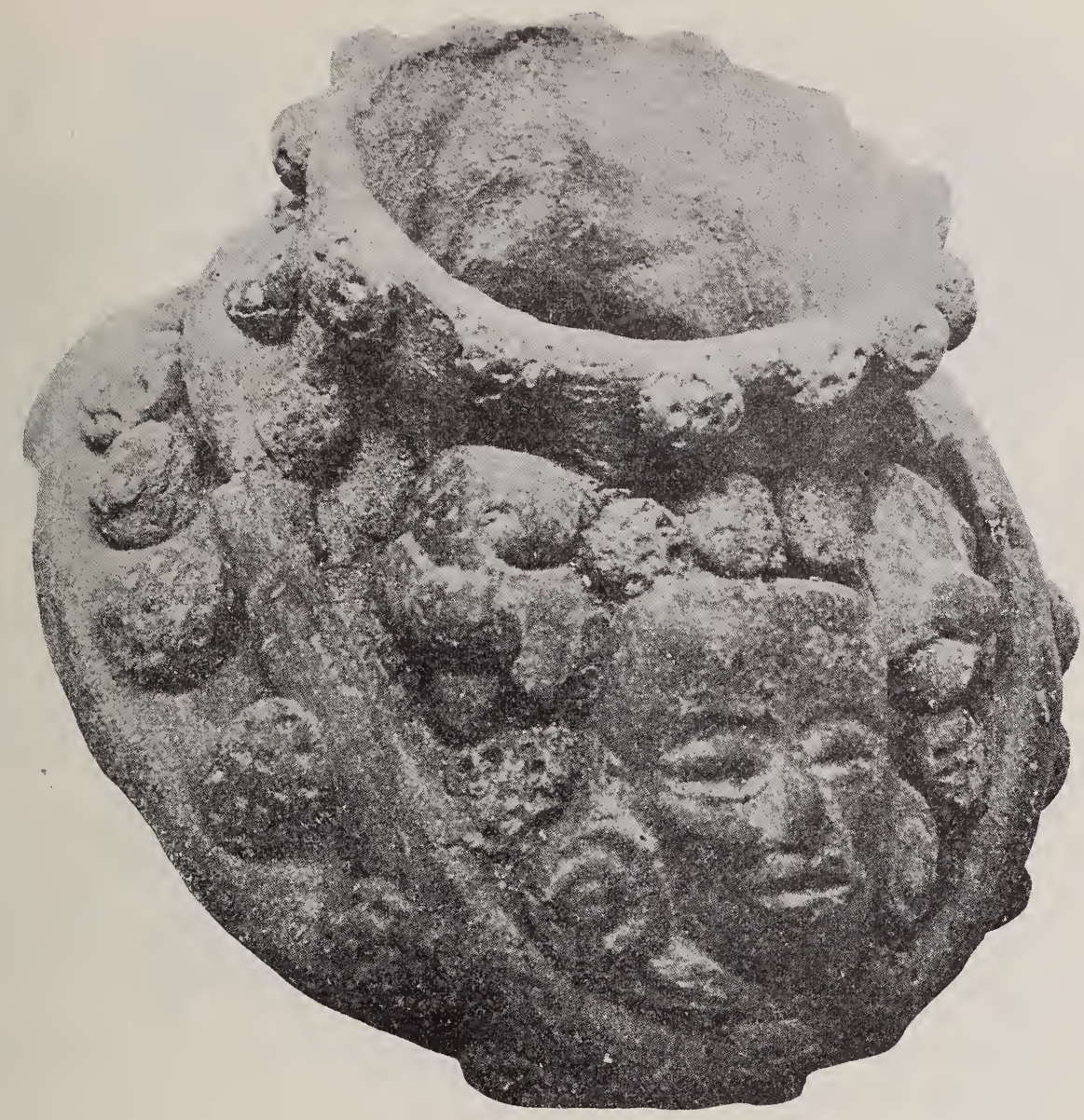

(Fig. 5.)

entonces, casi a 1 kilómetro al Oriente de Azcapotzalco; a poco más de 1 kilometro al Oriente de Tlilhuaca; inundaban Popotla; quedaban a 1 kilómetro Oriente de Amantla y algo más de 2 hacia el mismo rumbo, de Ahuizoctla; invadían porción de Tacubaya y Chapultepec; lamían Culhuacán y Mixcoac y Churubusco y se aproximaban por Tlacopaque y Coyoacán y Tenamitla (San Angel) al Pedregal.

Cuanto a Ríos, el de Tlalnepantla, tenía casi su curso actual; el de Los Remedios, su porción norponiénte, como en una tercia parte, ocupado por el Lago, y el de San Joaquín era ocupado en su mitad oriental.

La exploración nos ha enseñado un Río desaparecido y que corría de Norte a Sur, pasando al Poniente de Tlilhuaca y de Ahuizoctla, en donde volteaba hacia el Poniente, pasando al N. de Tepalcatitla y de Amantla. - No cabe dudar que era el actual de Naucalpan. Pues bien, ese cauce antiguo de arena y grava, constituye en parte y acompaña en otras, 
a los yacimientos del material arqueológico recojido y que ilustra la Monograf'a presente; así se explica la presencia de grava y arena en los ejemp'ares. Cabe suponer la invasión de los pueblos tecpanecas principales por el paso de ese Río; destruyéndolos. Hasta hay figurillas pulia as por el agua y que perdieron sus salientes por el rose, al ser arrastradas: vemos pues, que la Geografía se liga íntimamente con la Estratigrafía regional, misma que precisaremos al explicar la figura respectiva. Ahora, concluiremos con los nombres de lugar.

TLILHUACAN.-Lugar seco y negro: tliltic, negro; huacqui, seco y can, lugar. El sitio confirma la etimología, que hace referencia a la tierra:

AHUIZOCTLAN.-Lugar en el que hav barro o arcilla plástica. ahuic, a uno y otro lado; zoctli, barro, arcilla, tlan, lugar. Todavía, el lugar es de alfareros, y es abundancial en arcilla plástica. Erroneamente, y sin comprobación sobre el sitio se decía: Ahuexotla, lugar abundante en sauces, y también Ahuizotla, tierra del ahuizotl, especie de perro de agua (no existe en el lugar.)

AMANTLA.-Tierra o lugar de amantecatl, o sea los trabajadores en pluma. (Ni remotamente se conserva la industria en Amantla). Tal vez amantli, sea el nombre de las mantas indígenas tejidas con pluma.

AZCAPOTZALCO._-Lugar de hormigueros: azcatl, hormiga, azcaput zalli, hormiguero. Con efecto, abundan los hormigueros en el lugar. Torpemente se viene escribiendo Atzcapotzalco.

POPOTLAN.-Lugar del popotl, una graminea, de aplicación industrial. El sitio es célebre, por correr la tradición insostenible de haberse detenido Hernán Cortés, bajo el ahuehuete aún existente y que estuvo cerca de un pequeño teocalli a llorar la rota de la Noche Triste, del 30 de junio al 10. de julio de 1520 , cuando fué arrojado por los azteca y perseguido casi hasta Los Remedios, en donde reposó y recibió su desbaratado ejército.

TETETLAN.-Nombre de El Pedregal, célebre ahora, por el hallazgo del hombre más antiguo de América!

TENAMITLA.-Lugar de almenas o palacios. Ocupó el sitio del actual San Angel.

COYOHUACAN. - Lugar del Coyotl (canis latrans) coyotl co. yote, huacrui, flaro, en composición: huaca. Coyohuaca, y la n finai, de lugar. Sitio célebre por haber sido residencia de Cortés, al concluir la toma de Tenochtitla; ahí. quemó los pies a Cuauhtemoc y ahorcó a su primera esposa. Nada de ello ocurrió en el vetusto edificio del Palacio de Cortés, según recientes investigaciones de la Sra. Nuttal. edifirio construido con posterioridad, sino en el Barrio de la Concepción del mismo Coyuaran.

ATLACUIHUAYAN.-(Tacubaya).-Lugar en que se va por agua al Río: atlacuihua, ir a coier agua al río; yan. terminación verbal. El jeroglífco es un apilolli. o jarro para tomar agua. lleno de ella.

Existe también el jeroglífico de una mano coiien ${ }^{7} 0$ un atlatl, avarato de madera para arroiar dardos. la figura es del Códice de la Peregringción aztera y según la respetable autoridad de Orozco y Berra $\nabla$ don Fernando Ramírez, el nombre de lugar dice: donde se tomó o cojió 

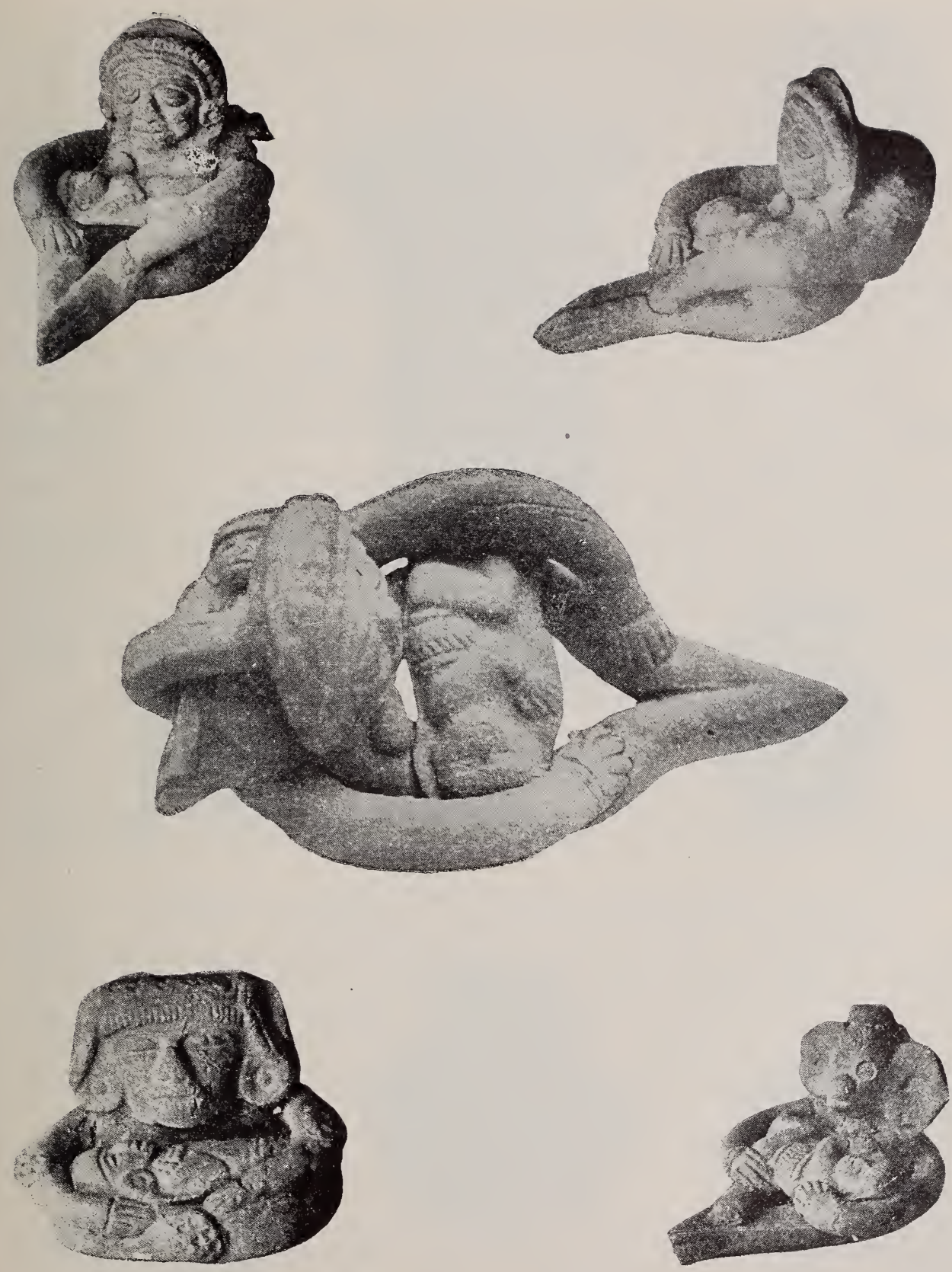

las MADRES. - LOS GOATES.

(Fig. 6.) 
el atlatl, dado que existe la tradición de haber los azteca, inventado el atlatl, en el sitio de referencia. El eminente Robelo, apunta en su Diccionario de Aztequismos, lo sorprendente que es el que con diversos elementos jeroglíficos, se forme una misma palabra ortológica.

Nosotros, "toda proporción guardada," creemos que el jeroglífico del atlatl, es puramente fonético, pues a ser representativo, tendría de conservar elementos de la palabra atlatl, el nombre de lugar, lo que no ocurre.

CULHUACAN.-Lugar de colhua, la tribu. Coltic, es torcido, y el jeroglífico, es un cerro de cima encorvada. También se da al lugar el nombre Teoculhuacan o Teocolhuacan, por el Dios Coltzin, a quien adoraba la tribu.

CACALCO.-(San Joaquín actual) fué lugar tecpaneca, perteneciente a Tlacopan. Lugar de cuervos: cacalli, cuervo, co, lugar.

TEPALCATITLAN.-Lugar de tepalcates (tiestería) o entre tepalcatl. Titlan, entre. Abundan, efectivamente los tepalcates. Cruztitlan. Palabra híbrida. Lugar de la Cruz.

ARMASTITLAN.-Lugar o río de las armas. Hibridismo con el que denominan los indígenas actuales el Río de los Remedios.

HUEYOTLI.-Camino viejo. Huehuetl, viejo otli, camino. Existe ya cubierto por el piso actual, un antiguo camino al Oriente de Tepalcatitla, Ahuizoctla y Amantla.

XALPAN.- (San Pedro. Lugar sobre arena. Xalli, arena icpan sobre. Está al N. de Tepalcatitla y entre el Río Armastitla y el antiguo cauce, por eso es arenoso.

Hablaremos a continuación de la Estratigrafía.

\section{ESTRATIGRAFIA.}

El estudio, descripción y clasificación de las capas en los terrenos explorados, constan en el esquema de la Figura 2 y las que le son anexas.

El proceso estratigráfico fué como se expresa en seguida:

En parcelas de 25,50 y 100 metros cuadrados, se roturó el terreno por fracciones de 15 metros de longitud por 5 de ancho, pues en tal forma, es fácil inspeccionar y aprovechar todo el yacimiento y el terrero, roturando con el pico y la pala, pero en tocando un yacimiento, únicamente el pico del Geólogo y pequeñas azadas sirven para continuar la labor; además, la tierra extraída, es pasada por criba luego que es arrojacia a la superficie, con lo que y por las reducidas dimensiones de la perforación, no se pierde detalle.

Los yacimientos en la capa o capas que comprenden, no son regulares, todo lo contrario, y es frecuente que aparezcan bajo forma de bolsones.

Hemos excavado desde la superficie, hasta 7 metros de profundidad; ésto solamente en lugares altos como San Juanico (el de Popotla); pero el máximum de profundidad ha sido de 5 metros 50 centímetros. Abajo de tales profundidades, aparece el suelo geológico.

Desde la superficie del suelo, de tierra vegetal, hasta $1 \mathrm{~m} .95$, encon- 

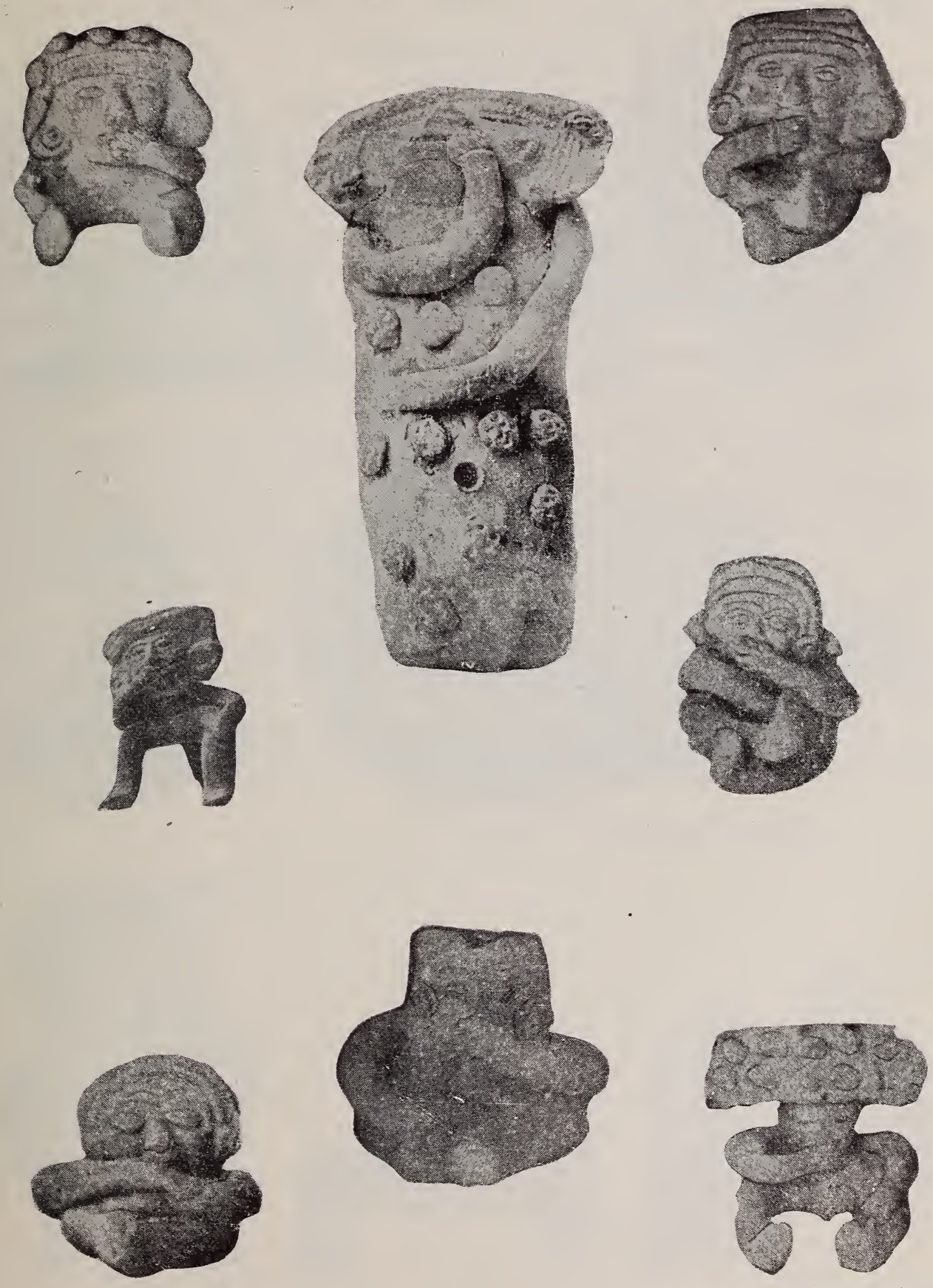

(Fig. 7.) 
tramos indefectiblemente, el tipo azteca, en Deidades, vasos, objetos de adorno personal y rituales.

De $1 \mathrm{~m} .95$ a $2 \mathrm{~m} .50$, el tipo teotihuacano.

De $2 \mathrm{~m} .50$ a $4 \mathrm{~m} .50$, el tipo tecpaneca.

$\mathrm{De}^{\mathrm{l}} 4 \mathrm{~m} .50$ a $5 \mathrm{~m} .50$ el tipo primitivo o arcaico.

En el estrato azteca hemos encontrado los incensarios (amacalli popochcomitl); los pisos de tezontle y cal bruñidos y restos de madera de techumbres, moldada en barro cocido; esquinas de piedra.

En el estrato teotihuacano, hemos encontrado los mismos pisos pintados, y pisos de adobe. Vasos negros característicos de Teotihuacán y otros de decoración raspada, cuentas de collar, de jadeita y orejeras negras en tubo también características.

En el estrato tecpaneca, el material arqueológico que ilustra esta Monografía.

Los porta-ofrendas (huencahuatli) vulgarmente denominados candeleros, los hemos encontrado indiferentemente en los estratos azteca y teotihuacano, con la misma factura e idéntico gálibo.

En la línea de separación del estrato tecpaneca con el teotihuacano, ha aparecido un vaso de uso desconocido, llevando sobre el borde tres chimeneas que no comunican con el interior, y una porción del labio, rebajada como si fuera vaso libatorio; siempre sin color ni bruñido, forma de cono truncado invertido y bien modelado, cocción incompleta, y el tipo humano en la extremidad libre de las chimeneas, es mofletudo, con tocado por pastillaje.

Inmediatamente abajo del estrato tecpaneca, está el primitivo, o arcaico, denominado "de los cerros" por la Escuela Internacional de Arqueología, aun cuando impropiamente, según lo hemos dicho en otras ocasiones.

Encontrar y fijar el horizonte o estrato TECPANECA, ha sido un triunfo del explorador William Niven y que debe de reputarse un gran descubrimiento arqueológico!

México, diciembre de 1919.

R. MENA. 

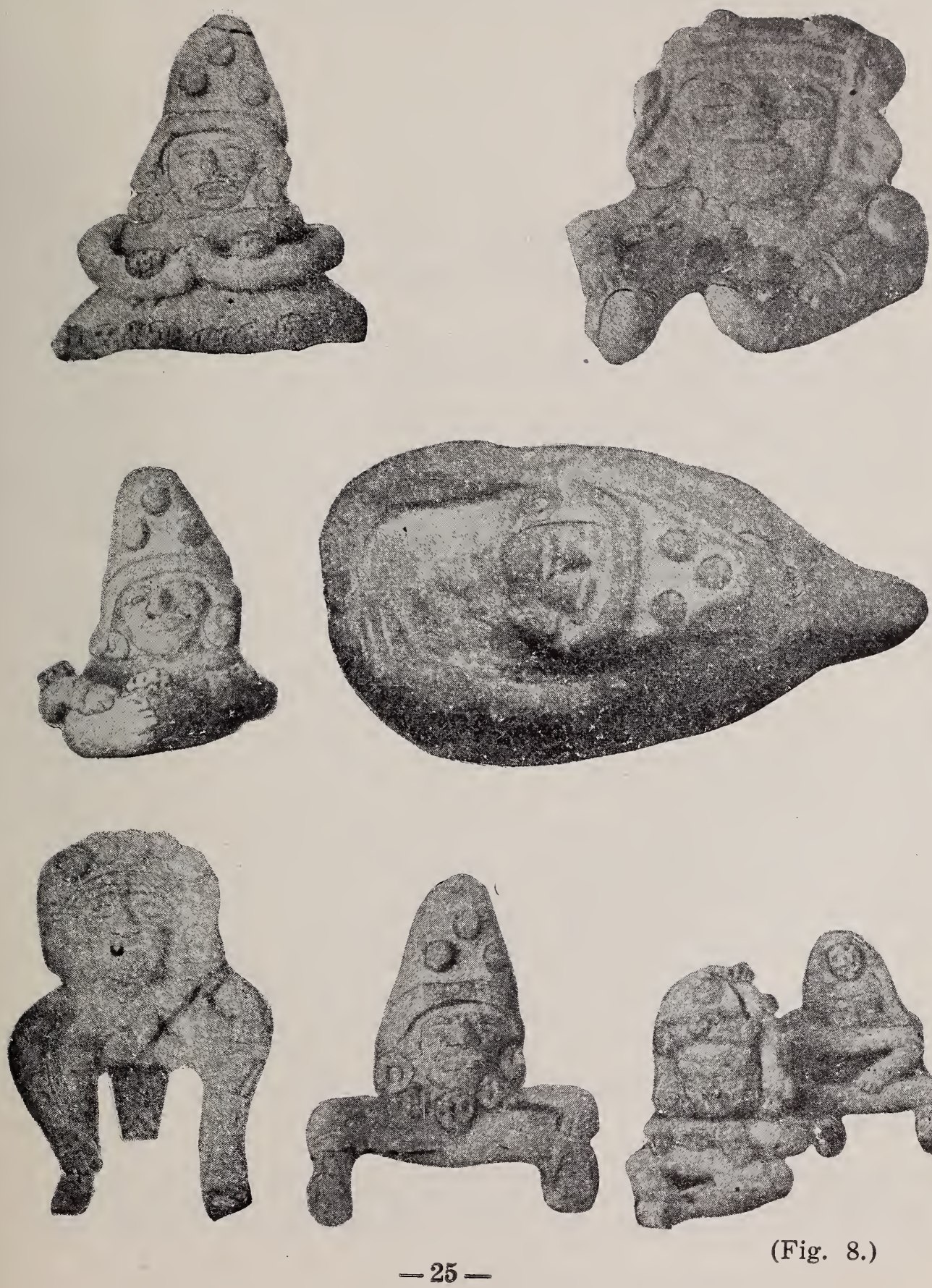

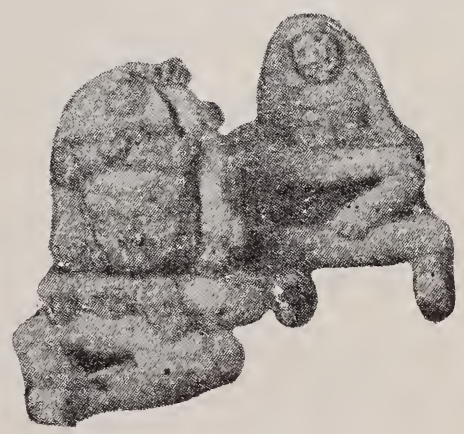

(Fig. 8.) 



\section{The Angient Tegpanecs in the Valley of Mexico}

The archaeological researches and explorations carried into effect by Professor William Niven during the years 1918-19 last past, in a zone lying in the northwestern portion of the valley of Mexico, have indeed proven most fruitful to the cause of antiquarian research as many interesting specimens of a prehistoric civilization have been found, and which serve most efficaciously to penetrate the mists and dissipate to a great extent the dense darkness which has here tofore enshrouded the social activities of our ancient ancestors.

By means of a constant and methodic system of explorations, places and districts have been thoroughly covered which include an area of territory absolutely TECPANECAN without doubt, such as SAN JUAN TLILHUACA, AZCAPOTZALCO, SANTIAGO AHUIZOCTLA, SAN MIGUEL AMANTLA, TACUBA, (TLACOPAN), SAN JUANICO, POPOTLA and SAN JOAQUIN (CACALCO).

The tecpanecan territory in the valley of Mexico, according to the fixed opinions as shared by all the early historians, as well as the pictographs as shown by the Codicils, embraced the districts of Azcapotzalco, Tacuba, a portion of Tacubaya, Coyoacán, Magdalena and Culhuacán; and this uniformity of ideas on the part of the various writers has been fully proven to he correct by the abundance or archaeological material discovered, and therefore the geographical situation of the tecpanecan settlements in the valley of Mexico can now be definitely determined. The accompanying map shows all the details with sufficient clearness, and defines the extent of the relations existing between the tecpanecs and the dwellers in the old lava fields.

As regards the system of exploration observed, properly speaking, it was made at all times along the lines of a stratigraphic method which experience had shown to Mr. Niven to be the correct one after years of research work in the valley of Mexico, with the view of determining the respective archaeological horizons with greater exactness than has been the case with the International School of Archaelogy, in 1911-12.

See our stratigraphic representation. We annotate thereon for the first time the "tecpanecan horizon", and which is unquestionably of a period prior to the one enshrouding the mysteries of "Teotihuacan." 
The importance of the tecpanecan horizon is of such a nature as to forge the link which connects the prehistoric or archaic era of the dwellers in the old lava fields, with the "teotihuacan" period, and thus we again confirm the opinions handed down by historians, by means of the archaeological discovery, to the effect that the "teotihuacan" civilization began to flourish with the dedication of the celebrated pyramids in the VII seventh century of the Christian era; and at that time, there is no doubt whatever but that a long time had elapsed, perhaps centuries had passed by, since the tecpanecan peoples had taken up their abode in the valley of Mexico with the Capital of their kingdom located in Azcapotzalco. Furthermore by considering the information which we have plucked, as it were, from the Chronicle of the Chronicles as contained in the History written by Orozco y Berra,- I repeat, by considering, taking into account the fact that when Torquemada was writing his "Indian Monarchy" about the year 1595, or before, the tecpanecan peoples claimed to have been residing in and around the environs of Azcapotzalco for 1565 years, it thus results that they had established their Capital city about the year 30 of the Christian era; and then when we realize that in order for them to perfect a norganization as a tribe and become so unified as to be able to constitute a stable form of government and establish their Capital, we see that this would not only require a period of thirty years but perhaps cover a whole century; so thus it becomes quite evident to us that the tecpanecs must have been dwelling in the valley of Mexico, previous to the birth of Christ. At all events, these are the conclusions formed in view of the discoveries made and referred to in this monograph, and the quantity and quality of the archaeological material which has been collected certainly serves to prove the correctness of same.

\section{THE ORIGING OF THE TECPANECS.}

The pre-Cortesian Codicil recording the Aztec Peregrination tells us that they were one of the tribes belonging to the great "Nahua" family who formerly had their abodein "Chicomoztoc" and afterwards peregrinated towards the valley of Mexico. This same authority gives us the hieroglyphic name, phonetically figurative, as: a "tecpatl (hard fossil) with which we can definitely and graphically portray the word as TECPANEC; but there have not been those lacking who have written the word as "tepanec", signifying a people with palaces, which alters considerably the correct application of same.

"Tecpatl" means a hard fossil, and "necatl" is the termination of a word applied in connection with the names of tribes ending in pa or tl; so therefore we have the word "tecpanecatl" signifying the people of the "hard fossil" of the people of "tecpatl" (place). According to the Codicil, and also to the historians, the tecpanecan tribes abandoned their dwelling place in "Chicomoztoc" in three sections, and arrived in this valley a long time before the coming of the Aztecs; but during their 

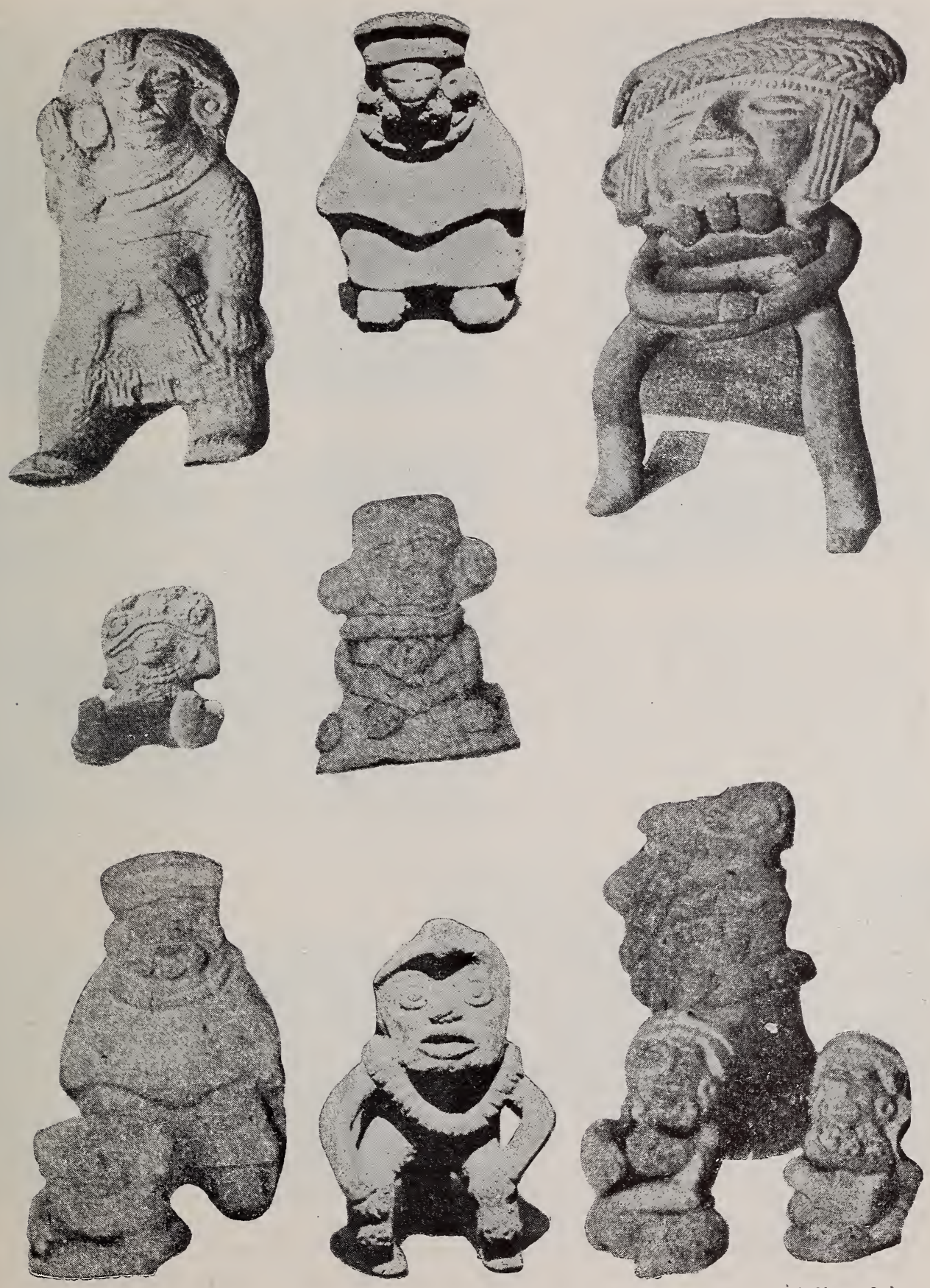

(Fig. 9.) 
several journeys into other lands they became divided into different groups or parties, and so it occurs that even according to the traditions of their own kingdom, they appear to have arrived at different periods: the first ones who made their appearance at a very early date were those of Azcapotzalco, and later came the tribes of Tlacopan. As the tribes or different fragments of the "nahuatl" race brought along with them their deities, we are of the opinion that the one known as "Tlacatecpanecatl" pertained to the "tecpanecs", and the archaeological material collected shows after study that when the division among the tribes took place, they had already an established religion and were performing their acts of divine service in accordance with the tenets of same; furthermore, we can see that they possessed considerable cosmogonical knowledge without doubt, as is evident from the fact that the various "nahua" tribes, even when on the march, always celebrated the festivals corresponding to the cycle of the seasons; and we find the Toltec tribes who belonged to the same etnographical and linguistical familiy, reforming the calendar 133 years before the dating of the Christian era.

With reference to the "tecpanecan" types, we will talre up this subject later, after having finished with a study of the archaeological material which we have in hand.

\section{ARCHAEOLOGICAL MATERIAL}

From the month of July 1918 to the end of the year 1919. owing to the many discoveries of archaeological obiects made bv my friend ant associate in study, Mr. Niven, my attention was very strongly attracted to these explorations on account of the rare speciments found that were heretofore unknown, as well as their peruliar manifacture. and the renth at which they were located in the subterranean beds lying beneath the surface of the valley. The first one consisted of an oval-shaped piece, without paint or coloring, and on the reverse side about half way rown there extended around a branch which formed an angle with the front part and which combination seemed to serve as the representation of a church desk or reading stand. On describing this figure we have stated that it refers to the irtes of "Xochiquetral" and "Chicomevochitl" nres?nting the floral offering ("xorhimeratl") the corr or snirjtral font in accordance with the ceremonials. and the worshin of the divers dejties of the "Flower days." We observed then the attitudes of the miniature figures revealing entire body, ant n'aced sidewiso s'rs to comparinate with the festivals of the cvrle of the seasons. and we became aware of the perfectness of the modeling and the style of headeear in con ${ }^{2}$ rats with the extremities of the human figures: and also the bulhs of the fiowers, as well as the cord imperfectly dono in a verv nrimitive manner. However we arrived at the conclusion that the tvpes were anthropologically tecpanecan, in view of stratigranhical evidences. and that the specimen was found at a depth of a little over three moters embedded in a stratum of sand mixed with ashes, mud-brick and stone dust composing the remains of fallen walls or the floors of dwellinos or temples. This valuable specimen to which we refer, measures 22 by 15 

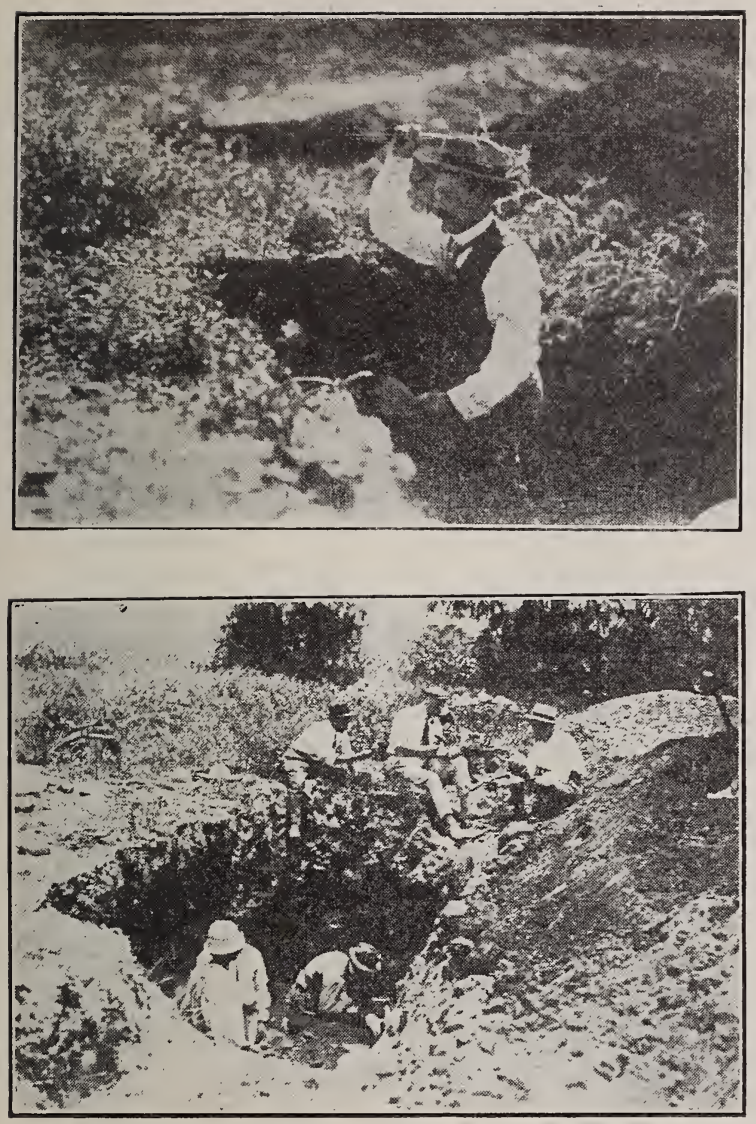

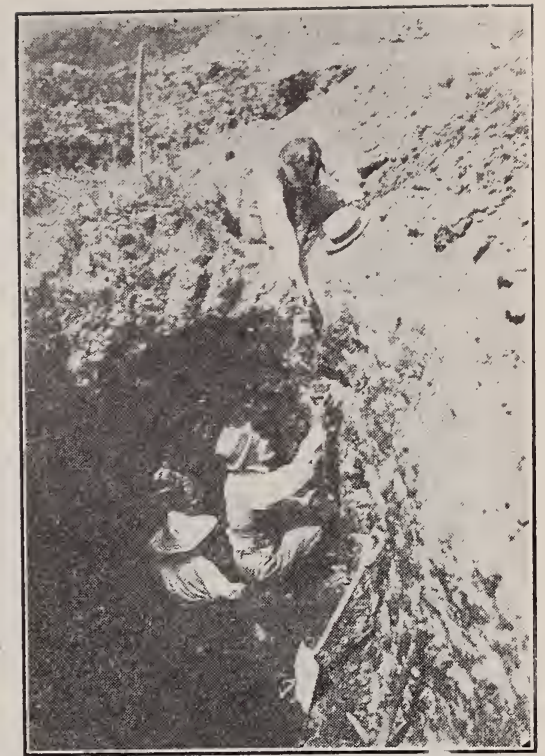

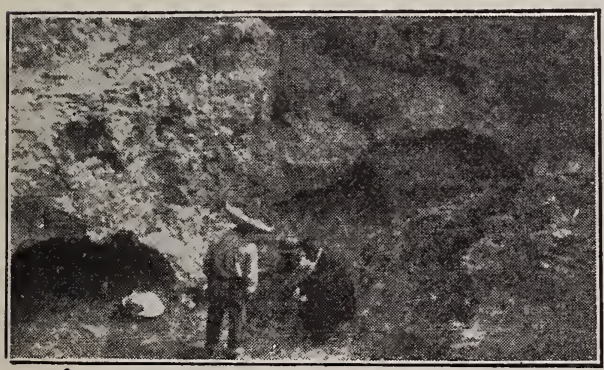

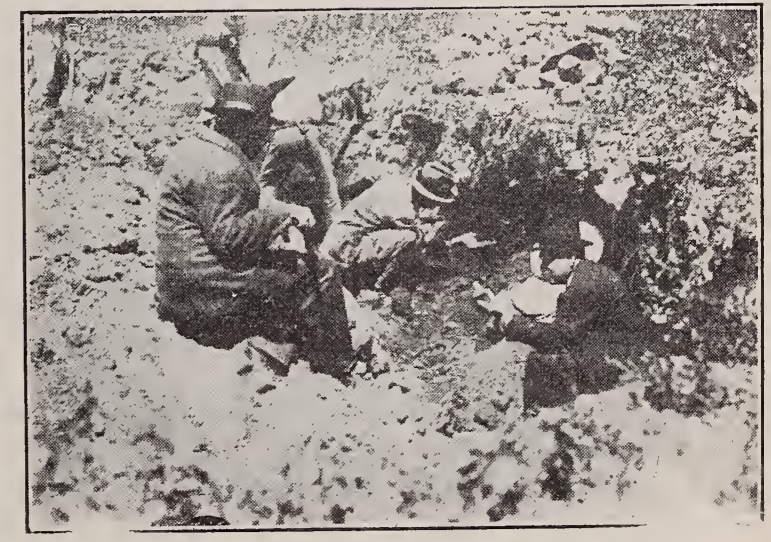

(Fig. 10.) 
centimeters. Its elaboration has been incomplete, although the exterior is smooth and evenly distributed. The stratigraphical horizon as revealed by the discovery is seen to be "tecpanecan" at a second period. The general technic observed shows applications in adhesive pastile work: the floral cord, flowers, vases and anthropomorphic figures having been modeled separately and attached in a crude manner... so unique and peculiar in their class: the miniature heads and bodies have been cast in molds, (Figure 3.), and proceed from Santiago Ahuizoctla in the Azcapotzalco district.

From this same place, and in a similar underground stratum as well as at the same depth were found the vases as per the Figures numbered 4 and 5. The No. 4 is of the "apaztli" order, 16 centimeters high and 24 centimeters in diameter, adorned with the floral chain or cord, and with sufficient space left in the belly of the vessel as to permit the grouping therein of miniature figures of night-birds, and the deities of the "Floral days" as was the custom, all placed and attached thereabouts by means of adhesive pastiles. The heads of the deities appear with eyeshades and which are archaic representations of "Xochipilli," the god of fire and flowers. The "tlacatecolotl" reveals the belly part as ornamented with flowers.

Figure 5 is a vase of the "comitl" type proceeding from the same place as before mentioned, as well as from the same stratum and found lying also at the same depth as the other specimens hereinbefore described. The workmanship is not even and imperfectly finished; the decoration is of the "xochimecatl" style, and encircling the belly appears the mould of a face surrounded by flowers of different kinds; of a primitive nature which in this instance we will call aztec; the flowers numbering seven and to which we apply the word "Chicomexochitl" the masculine god of "Xochiquetzal," with a height reaching 13 centimeters, and in diameter 7 centimeters. In this stratum, the same as in the other one, besides the objects discovered, there existed considerable gravel.

With reference to the "Chicomexochitl" we cannot fail to notice the pronounced racial type, with the long, oval face, high smooth forehad indented, as it were, in the center along the hair line. In fact, the little head was distinctly tecpanecan and pertaining to one of the three periods embraced in this most interesting culture.

Figure 6 represents a group of mothers with their babies. The manner in which they are carried forms a veritable, ethnographical document, the one marked with the letter A, a, carries twins, - one twin is being held in the arms, and the other one is carried on the back. The mother is entirely nude revealing her breasts; the headdress consists of bands of cloth through which the hair protrudes and hangs along the sides as disk-shaped ear coverings. Proceeding from out the back part of this headdress as mentioned, there appears a large, heavy band of material which extends down connecting with the waistband, wihch forms a space or place appropriate for the carrying of one of the twin babies.

The mother's countenance shows much expression; the eyelids are turned downwards in contemplation of the child, and the mouth reveais a lingering smile peculiar to the mongoloid racial type. 
As we find takes place in this culture, the extremities are not well designed, and in fact are badly modeled,-a characteristic which does not occur in the head work; however, the same conditions do not obtain in the technics of the children.

The $\mathrm{B}$, does not represent a living woman, but a goddess recognized by the flowers garlanding the arms and feet, and who carries in her arms not a live child, but a household god.

The $\mathrm{C}$, is a small representation of the face-mask of "Xochipilli" with a nose-ring consisting of a spheroidal flower and large discoidal ear coverings, as well as a head ornament in the form of a flower (Is this "Xochiquetzal?). At all events, it pertains to a period previous to $\mathrm{A}$ and $\mathrm{B}$.

It is surprising to note by the headgears, ear coverings and ventral bands around the infants, that they continued in vogue up to the aztec period in the time of the Spanish conqueror Hernán Cortés.

Figure A, is 6 centimeters in height, by 6 in diameter measured from arm to arm, and 11 centimeters in length.

Figure B, is 6 centimeters in height, by 7 centimeters in width, with a length of only 5 centimeters, as the legs are folded inwards.

Figure $\mathrm{C}$, is 5 centimeters high, by $5-1 \mid 2$ centimeters in width, and $7-1 \mid 2$ centimeters in length.

The figures are all made of a fine clay material, of a yellowish color, and incompletely baked.

They all proceed from the subterranean stratums discovered in San Juan Tlilhuaca on the dividing line between Atzcapotzalco an the State of Mexico, and found embedded at a depth of between 4 and 4-1/2 meters beneath the surface in a layer of gravel. S? $\mathrm{n}^{\mathrm{d}}$ and ashes The interior parts of the arms and legs were found filled with tho sant and fine gravel, with the sand alone adhering greatly to the hollows and projecting parts of the face.

\section{SECRET RITES.}

Figure 7, represents a group of these lit.te human imag's nortaining distinctly to the tecpaneran stratigraphic horizon. foun here and there in the districts of Tlilhuaca. S`n Juaniro. A mantla an 1 Ahuizoctla, as well as in Tepalcatitla, a nlace situater botween A man'la and Ahuizoctla,- and in every one of these locations the romnosition of the underground stratum was just the same. consistino of sant and gravel. I accompanied Mr. Niven to the places where the excavations were being made, and I can assist the discoverer iust mentioned, to the extent of being able to testify as to the authenticity of these subterranean deposits, untouched until now,-as well as the interesting specimens being brought to light from out the subsoil of this ancient valley which will so enrich the archives of American archaeologv.

The general characteristies of these little figures are similar to the ones before described; floral representations abound in all the styles of headdress, as well as in the neck ornaments, and it is very notable to see that in all these little images, whether masculine or 
feminine, of deities or of human beings, they always show one hand as pressed to the lips, and the other one resting on a muscle. At other times, the hand is held against the breast. And in connection with this sign which still prevails in modern secret rites as has been transmitted down the centuries from remotest ages, it cannot be said that it is strange that same exised among the tecpanecans during the solemn and mysterious rites dedicated to the gods of the flowers and fire. It seems wonderful to discover here in this horizon, the old and venerable god, Huehueteotl, the most ancient of the American pantheon, whose face is crossed and recrossed by the lines and furrows of age,-and the little figure of this god appears in the attitude of making.. the secret. sign...!!

And what is to be said of "Xochiquetzal" which from the earliest periods of tecpanecan history, appears with the sacred "quetzal,"-that worshiped symbol, of the compressed crest and brilliant plumage, of the ancient races, iridescent on the headdress, and with a necklace of flowers encircling below? In our figures mentioned, this sacred bird, "quetzal" shows the beak as broken; and the rest of the head adorment is similar to that of "Xochiquetzal" and "Xochipilli," but of fine execution, design and special style; on the other hand, that is to say with reference to the neck ornaments and flowers, it is readily seen that they are of primitive fashion, and the body is substituted by a very poorly modeled cone and with applications effected by means of adhesive pastiles in representation of cords and flowers; and furthermore, the goddess has assumed a mysterious... secret attitude. Neither is this all: there are miniature archaic figures pertaining to the first period of the tecpanecs, who without doubt were contemporaneuos with the ancient inhabitants of the old lava fields, and with whom they must have been in contact, (perhaps ten thousand years ago, - - which reveal the ritualistic attitudes to which we have referred,-and these miniature human figures, both masculine and feminine, in these secret mysteries, show the genitai organs in a perfectly nude condition as if such rites had some relationship with those of phallicism, as will be mentioned later.

\section{PHALLICISM OR THE WORSHIP OF THE PHALLUS.}

The group represented by the Figure 8, shows the conic form of headdress with floral ornamentations. From ancient history we have known that the conic form of headdress has had some connection to the phallic rites, or at least this occurred among the aztecs and totonacs, although they were not the only ones addicted to phallic worship, in view of the fact that we know that this practice was current and in vogue, (as proven by old manuscripts,) among the "quichés-mayas" and the tarascan races. So now it occurs that we find that there existed an analagous system of worship as practiced by the tecpanecs in the earliest periods; in fact, a large portion of the human figures with the conic form of headdress, exhibit the male organ and even the feminine figures - with identical headdress-also reveal the pudendum or vulva with every detail; and here is something more.... wonderful.... There 

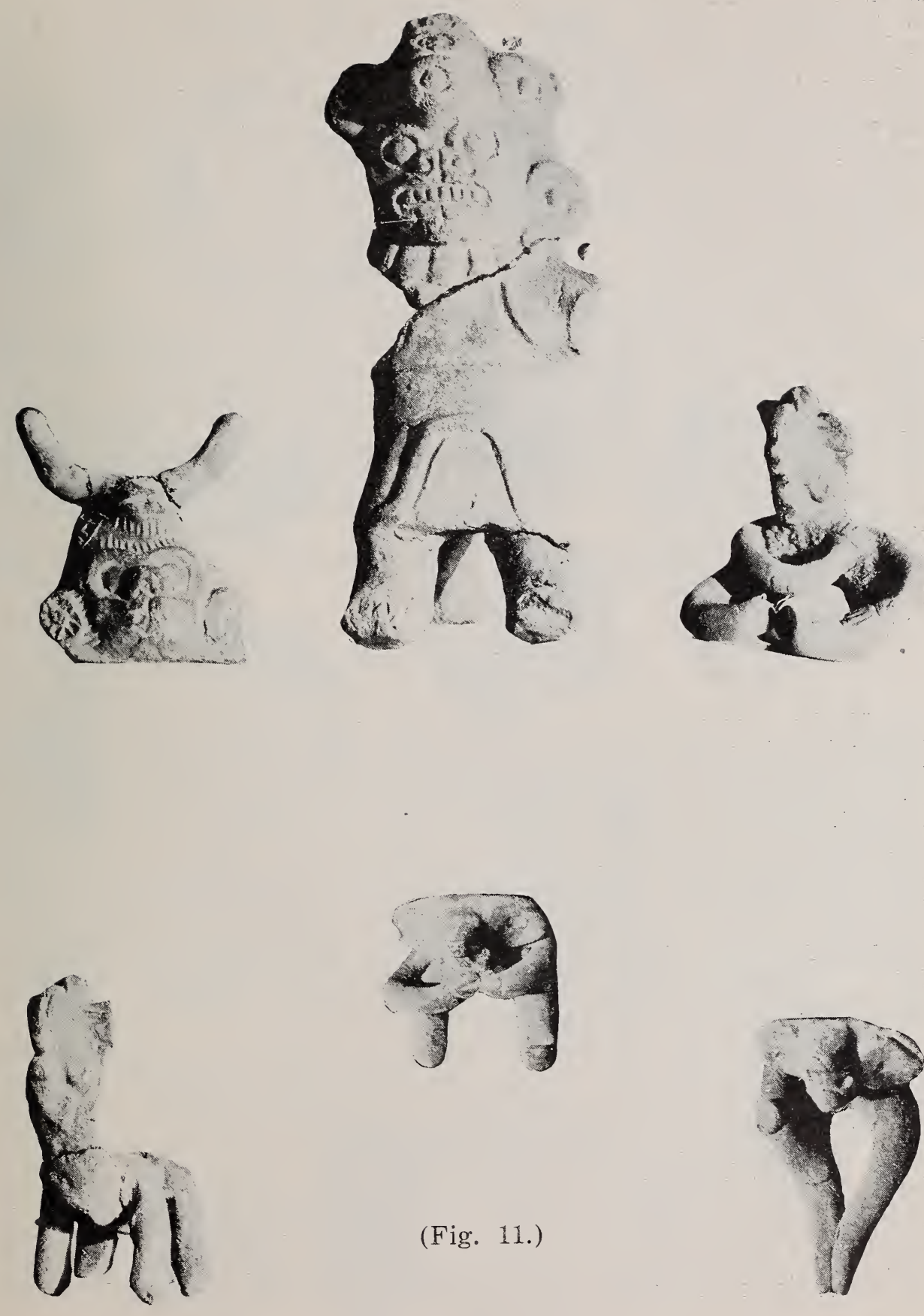

(Fig. 11.)

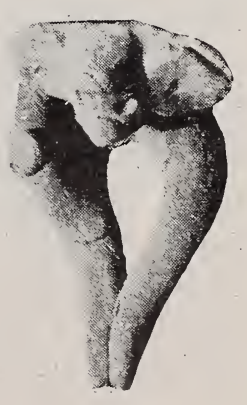


is an object which seems to be falling, which at first sight appears to represent a small timbrel, but on closer inspection it is seen to be the representation of a drop of semen oozing from the deminine organ of generation. In fact, in the Jonuta work of relief (No. 26 in the Gallery of Monoliths in the National Museum of Archaelogy) the group of symbols of the male organ of generation which are portrayed in the manner of projecting cloves around the head of the priest of the temple, are evidently ejecting semen, and thus the seminal fluid is seen to be falling in ovoid drops in a form very similar to the specimen which we have just described. Given the importance of the semianl production in the matter of generation, it is congruent to conjecture and form opinions regarding the deity of which it is an attribute.

The relation existing between the phallus and the principle of generation was established in the tecpanecan period, and yet survived throughout the Aztec pantheon, in the "Coatlicue," for example, in the Milky Way; Iztacmixcoatl where took place the worship and the representation of serpents among the Aztecs, were all due to a phallic origin, neither more nor less, as initiated among the tecpanecs as demonstrated by the specimens examined. They are of identical general characteristics as the preceding ones, and their dimensions vary from between... and... centimeters.

They were found in Tlilhuaca, in the stratum as described, and with the sand tenaciously adhesive.

\section{OTHER HUMAN FIGURES.}

We have two types of men and one of a woman represented by Figure No. 9; with reference to the first mentioned, the A, is notable for the headdress arrangement, as well as for an additional piece in the form of a church desk or stand which serves as a support; the hair shows as being trimmed in the form of a ringlet over the forehead, leaving two large locks of hair that hang down past the ears, and not interfering with the diskshaped ear coverings as have been previously mentioned. Palm leaf ribbons or bands encircle the head; as regards the face portion the nose has been considerably worn owing to the destroying effects of external agencies,-and the necklace is a representation of three flowers. and the whole piece has a height of 14 centimeters. The face shows as having been prepared by a molder.

The $B$, has been cast into two different parts: one for the head and right hand, and the other for the rest of the body. The expression on the face, and the hieratic nature of the specimen together with the tecpanecan type, is most surprising,-and cushions of flowers dot here and there the hair dressing as it hangs below on the left side. The bust is nude, and the arms and legs have their floral coverings. Across the breast extends a garland of flowers, and then appears a marvelous "maxtlaltl" resplendent and imposing as the turrets of a fortress: besides, there is a belt at the waist exhibiting the elegant knotted cord so typical of the deities of the gods of fire, wohse countenance it bears. The little 

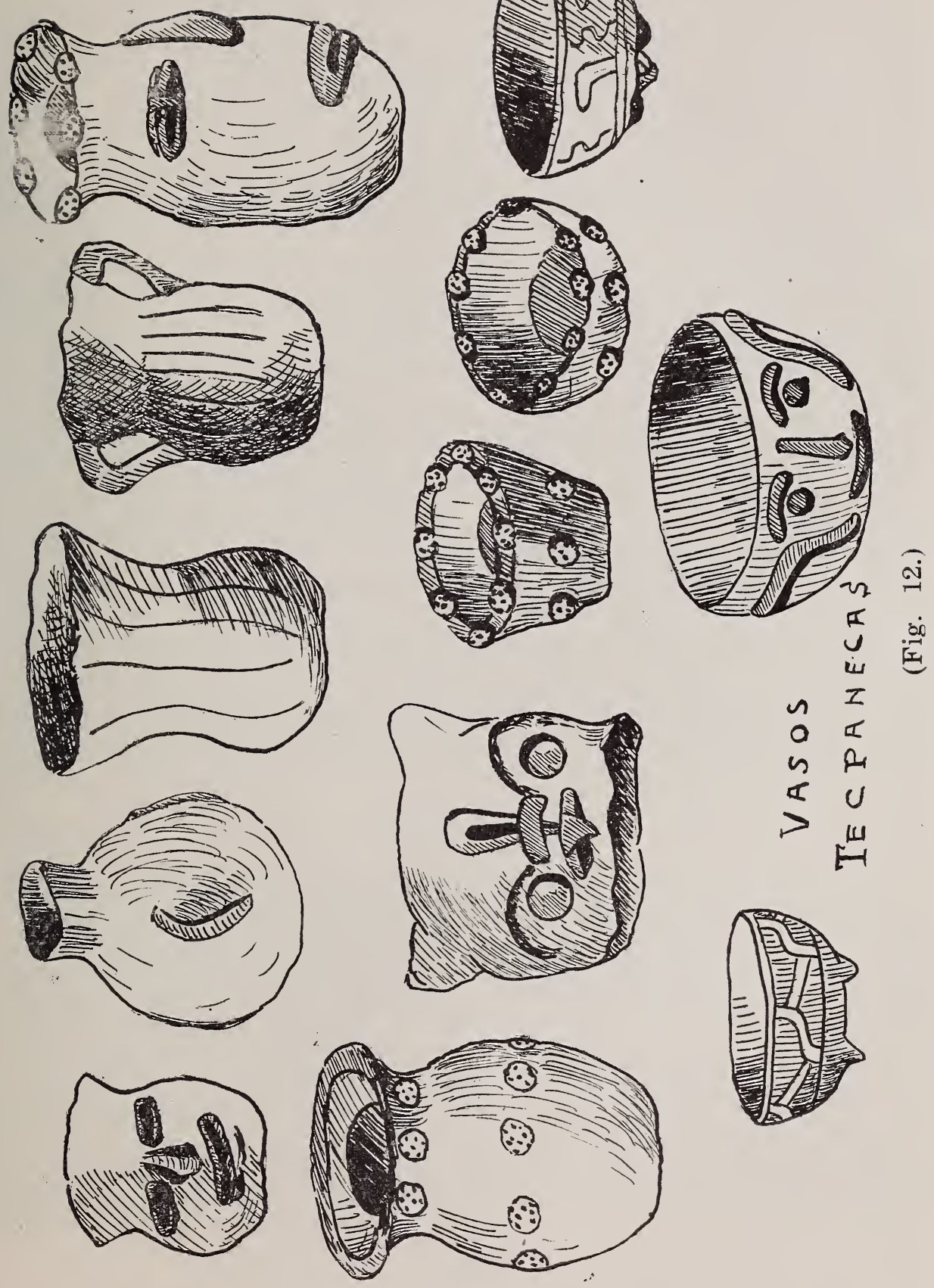
figure is 15 centimeters in height by 7 centimeters in width, and the back part of the facial portion is smooth and even.

With $\mathrm{C}$, we have marked a womanish figure 11 centimeters in height by 7 centimeters in width. The countenance resembles very closely the type and technic in modeling, as those of the lava field dwellers; the head costume is almost oriental in design and characteristically tecpanecan; and with reference to the clothing, the "huipilli" (shirt) and the "cueitl" (skirt) are eminently of a distinct buddhistic nature. This style of dress: with the various parts adorned with ribbons, bands, and rich designs subsisted up to the time of the Aztecs, and we find it in vogue among the inhabitants of "teoticuacan" during the building of the pyramids, who as we have before demonstrated, flourished at a much later date than the tecpanecs.

Notwithstanding the remoteness of the ancient period to which this little figure belongs, it has a collar piece with ornamentation consisting of three flowers, and below there appears two necklaces with five beads in each one: four small ones, two at each end, and a long one in the center. The number 5 ("macuilli") may have a reference to "Macuilxochitl."

It is convenient to here observe that neither one of these three miniature figures represents in itself any one deity. They are persons adorned in the fashion of the deities, and this womanish one, no doubt was a household goddess,-because on examination it is seen that the lower portion of the posterior part of the face has a small pivot which serves to sustain same in a vertical position.

There are other miniature figures, representing generally household gods: the head is well modeled with the head costumes corresponding to their category, and the body is invariably without arms and legs resembling considerably the form of a truncated pyramid with its greater base reversed. These bodies are evenly elaborated, and with smooth lines showing the points of insertion for the arms and muscles; and also reveal on the front portions a series of graphic diagrams and tracings. They are molded in one piece.

\section{THE MOLDS.}

The molds for heads, bodies, household gods, breadmaking rollers, showing whole body,-are heavy and well baked, and it is very evident that they were constructed of the very best clay material, highly pulverized and beaten into shape while yet fresh, on a model which had been most carefully fashioned. The use of the mold was as absolute necessity imposed by the cult of the deities, and this accounts for the large number of household gods and heads of deities so modeled. Furthermore the art of taking familiar portraits was practiced,-at least, the types desired were copied by the tecpanecan sculptor among the kinsmen, and which is the only explanation to be offered when it is seen that the deities all show the same characteristic racial type, notwithstanding the use of elaborate head costumes, ear coverings and collar pieces, which of course altered to some extent the physiognomy. 
By means of these molds, they cast their figures and images by applying a great pressure around the plastic clay to give the perfect form which they knew so well, and then leaving it to dry in the sun within the mold; thus after the water had evaporated from the earthy material, the molded form began to loosen, whereupon it was removed to the shade where same was subjected to the baking process over a wool fire, or inside of rudely prepared ovens which system was hardly known to them. The heat was not directly applied in the second instance, because we know this from the fact that in the tecpanecan horizon we have found some very small pieces resembling the bottom of a vase with three feet attached. The object was passed on down to the Aztecs, and even in this XX century the native Indian potters use this method for placing their ceramic products inside the oven in such a manner as to prevent the fire from exercising a direct heat over them, and this process is still called.... in the Aztec or Mexican tongue, but commonly known in Spanish as "caballitos" (little horses).

We do not find that the tecpanecs ever molded any of their works in relief, to apply them afterwards to their vases, urns, and incense burners by the system of adhesive pastiles; at least this technic peculiar to the Aztec and Teotihuacan periods has not been discovered in any of our explorations effected up to the present time in the stratigraphical horizon of the tecpanecan peoples.

The mold used for the manufacture of the bread-dough roller: consists only of a hollow portion necessary for forming the body of the instrument, as the handles on the sides are prepared otherwise and inserted by adhesive methods, previous to the baking process.

\section{SORCERERS or MYTHOLOGHCAL BEASTS.}

Figure 11 is a very remarkable specimen showing two human subjects in molding having a head resembling probably that of a monkey and with such a peculiar headgear, that by inverting the figure owing to the adjustment of the headtire just referred to, and the way the eyes are placed, the face remains the same as if no reverse movement had been effected. There is also one, in which the body appearing to be seated when in its normal position, turns out to be performing an acrobatic feat, upon inversion, with the head in the sams position as before stated, and this is of the most primitive design.

Heads of the same humanized types, so to speak; are found attached to bodies of quadrupeds, and even some of them have the crest of "Macuilxochitl." What is this? The specimens are authentic ones discovered in the subterranean beds and places as described at the beginning of this monograph.

When it is realized that from the remotest times of antiquity, there existed among the tecpanecan peoples many peculiar rites, and an interesting and extensive cult based on cosmogonical principles,-it is not out of place to consider that there must have existed sorcerers or necromancers who changed the aspect of their bodies, and transformed themselves completely, with frequency. At any rate, the interest in these specimens becomes very great. 


\section{VASES.}

Everything, in this interesting culture as analyzed, is a wonderful revelacor: the vases are by no means lacking in this feature, and especially inose corresponaing to the first period so exclusively consecrated to the worship of the noral deities, are plethoric with floral adornments, boch around the moun

With reference to the morphology of the subject, the form in (?)

,badiy modeled, without decorated edges, and with floral applications, are coninued in the structural arrangement of the jar ("comitl") wich well defined lines and proportions, but of a rudimentary style of modeling, with applications attached by means of adhesive pasciles. There exists the tenuency 10 reproauce the human form, as well as the forms of ra+ious aninals on these vases, especially in the (?).....; thus we have the represencation of the night-birds (resembling owls) and the face of a man, all attachea by the aahesive pastiles to the belly of the vases. Mention shouia here be made that the vases referred to, are frequenuly very small, and appear to be mere playthings, or perhaps, simply patterns.

Fs pertaining to another period, we find the form "xicalli" with heavy pas.ie appiications adorming the belly portion; also the same fo.m wich feet extenaing from the upper edge of the object to the bucom; 3 tee, and even 4 alternacing in this way with frequency: two extenaing aown from the upper rim, and two attached to the botiom. "This form does not periain to the "nahua" civilization but to the "Larascan," and perhaps are to be considered as souvenirs of the transit of the "nahua" tribes across the territories of Mihuacan. Even the $\mathrm{g}_{1}$ aphic decorations, the free hand tracings, together with the zig-zag lines or little circles of diverging rays, recall to memory the primitive nature and the ingenuity displayed in the technics of the early periods. (Megaliths of the Lava Beds.)

Among the vases shown in Figure 12, there is a ventral decoration which is hollowed work, - a style which is seen to have subsisted during the "teotihuacan" epoch, and showings symmetry and elegance in its sobriety of detail.

Comparing this figure with numbers 4 and 5, we can appreciate the morphological evolution of vase manufacture as performed by the tecpanecs. As regards the coloring, we have not fuond a similar one on any vases pertainning to this culture,-but on the miniature human figures representing the god of fire, alternating red yellow bands or stripes appear thereon.

\section{THE EVOLUTION OF THE TECPANECAN TYPE.}

In the tecpanecan horizon, there begins to be defined an anthropological type which is "sui generis," and at the same time brings to memory the prehistoric epoch of the lava field dwellers; and as we ascend in the mentioned horizon, we find that owing to an improvement in the technic followed in the modeled forms, as well as in the art of portraiture, the features are considerably truer, and finer in detail as the art continued 
to be perfected; but the head which requires nothing but observation, fixes its silhouette in 3 periods: flat forehead and sunken on the top of the head.-High flat torehead, and also curved and bulging.

The first mentioned corresponds to the stratum lying at the greatest depth, and forming the link, as it were, with the lava dwellers' period.

The second and the third ones relieve as it were, and perpetuate so to speak the "teotihuacan" civilization as these types reached by transmission down through the ages to their dominios, - and we still tina them among the Aztecs in the Cortesian period of the XVI century. In this way can be explained the presence of the tecpanecan type found in "Teotihuacan," where the large quantity of Little Heads existing there, show the characteristic type as before stated. So thus we deduct that the cultural extension of the peoples of "Teotihuacan" did not reach the "tecpanecans" because it has been established that these existed with priority, and in their horizon there appears neither anthropological types, domestic ceramics, nor vestige of the "teotihuacan" ritual.

This is not the place for making a comparative study of the anthropological types of the seven "nahuatl" tribes; but we can say "a priori" that it would show a distinct setting as the result of habits, methods of alimentation, and other characteristics, undoubtedly.

With further reference to the type of the tecpanecs, we can affirm that from the period of their earliest history, when the sculptors applied by means of an imperfect system of aahesive pastiles, the eyes, nose, lips, and ornaments, - the anthropological characteristic of the head can be defined.

The tendency towards prognathism exists in the tecpanecan type, but only as a tendency; because a distinct and well-defined prognathism has not been found by us, neither in the first tecpanecan period, nor are the miniature heads of an orthognathous character.

The depression observed in the front part,--Has it been produced artificially? It so appears, although we are not in possesion of any creaneums of this type, in order to be able to prove such an assertion.

\section{GEOGRAPHY and REGIONAL STRATIGRAPHY.}

The tecpanecan geographical data is duly shown on the map accompanying Figure 1., and from it can be followed the evolution of the tecpanecs from the southeast to the northwest across the valley of Mexico,--and considering at the same time the relations which must have existed between them and the dwellers in the old lava fields of whom we have spoken, during their occupation and journey from the southland as they descended and advanced from out the mountain fastnesses of Ajusco, along the slopes adjacent to Mixcoac, Tacubaya, and San Joaquín; and finally arriving on the table-lands surrounding Azcapotzalco, the Capital of the kingdom whose hosts were to push the Aztecs back. toward the old lava, beds, and thence to the shores of the lake, where destiny had reserved for them the site for the founding of the great "TENOCHTITLAN." 
During the tecpanecan period, the hydrography of the region in question was by no means the same as we to-day know it: in the first place, the waters of Lake Tezcoco at that time reached a point within one kilometer to the east of Atzcapotzalco; a little over one kilometer to the east of Tlilhuaca, and inundating Popotla; the waters reached within one kilometer to the east of Amantla, and a little over 2 kilometer. in the same direction towards Ahuizoctla; they invaded the precincts, or say, a portion of Tacubaya and Chapultepec; they also bathed the lands adjacent to Mixcoac and Culhuacan, as well as Churubusco, and approached the territories of Tlacopaque, Coyoacán, and Tenamitla (San Angel) among the lava beds of Ajusco.

With reference to the rivers of the valley, the Tlalnepantla stream followed practically the same course as to-day; the Los Remedios in the northwestern portion and extending over about a third of the distance towards the lake, and the San Joaquín river flowed through the eastern half of the valley.

During our explorations we have discovered the course of an old river now disappeared, which flowed from north to south passing to the westward of Tlilhuaca and Ahuizoctla, and thence continuing in the same direction adjacent to the districts lying north of Tepalcatitla and Amantla. There is absolutely no doubt but that it is the same course known now as the Naucalpan. Well, it so happens that that old river bed of sand and gravel constitutes in part and accompanies at other times these stratums or subterranean deposits of the archaeological material collected, and treated of in this monograph. This explains, of course, the presence of sand and gravel in the specimens unearthed. It is very reasonable to suppose that the invasion of the waters of this river overflowing the principal tecpanecan settlements in the valley, simply carried everything before it, and destroyed them completely. There are even miniature figures polished by the action of the waters, losing their principal features by the friction of the movements produced when they ware hurled onwara and downward before the mad rush of the liquid element. Thus, we see that Geography is intimately connected with regional Stratigraphy, as demonstrated in the present instance, and which we will refer to in detail when describing the respective Figure. Just now, w'e will proceed to conclude with the suject relating to the names of places.

"TLILHUACAN."-A dry and black place: "tliltic". black;" "huacqui" dry, and "can" place. The site certainly confirms the etymology making reference to the earth.

"AHUIZOCTLAN" place where is potters' earth or plastic clay: "ahuic" on both sides; "zoctli" potters' earth, clay; "tlan" place. In fact, it is still a potters' quarry, and abounds in deposits of plastic clay.

Erroneously, and without a foundation for thus referring to the site in question, it has been called:

"Ahuexotla" place abounding in willows; also "Ahuizotla" land of the "ahuizotl" a species of water-dog. (not existing in the place.)

"AMANTLA."-Land or place of the "amantecatl," or the plumed workmen. (There is absolutely no record of the subsistence of this 
industry in Amantla.) Perhaps, "amantli" may mean the native blankets woven with feather work.

"AZCAPOTZALCO."--Place of the ant-hills: "azcatl" ant; "azcaputzalli" ant-hill. It is a fact that ant-hills are plentiful there. Thus the name should not be written as "Atzcapotzalco."

"POPOTLA."-Place of the "popotl," a graminieous product for industrial purposes. This site is made famous by the untenable tradition to the effect that Hernán Cortés stopped and shed bitter tears here under the old cypress tree which is still standing, and which was near to an Aztec temple, on the memorable "Sad Night" when his hosts fled before the victorious Aztec legions on or about of 1o. July of the year 1520, and were pursued to the vicinity of "Los Remedios" where he at last rested and gathered together his scattered army.

"TETETLAN."-Name of the "Pedregal" (Lava Fields) celebrated at present for the of the most ancient men of America.

"TENAMITLA."-Place of turrets or palaces. It occupied the site on which San Angel now stands.

"COYOHUACAN."-Place of the lean "Coyotl" (barking dog.) : "coyotl" coyote; "huacqui" lean, in composition: "huaca," "Coyohuaca," and the final "n," the place. This site being celebrated for having been the residence of Hernán Cortés after having taken the city of Tenochtitlan; and it is said that it was here that he tortured Cuauhtemoc by burming his feet, and also strangled his first wife to death. However nothing like this occurred in the old edifice of the Palace of Cortés, according to recent investigations made by Mrs. Nuttal, and which edifice was erected at a later date in the la Concepcion suburb of the same Coyuacan.

"ATLACUIHUAYAN."--(Tacubaya.) Place in the river to go for water: "atlacuihua" to go and draw water from the river; "yan"--a verbal termination. The hieroglyphic consists of a "apilloli" or water jug filled with the liquid.

There also exists an hieroglyphic of a hand seizing hold of an "atlatl" or wooden instrument with which to throw darts, and is a figure taken from the Codicil showing the Aztec Peregrination, which according to the respected opinion of Orozco y Berra, and Don Fernando Ramírez signifies a place meaning: place from whence the "atlatl" was taken or seized, and in view of the fact that there exists a tradition to the effect that the exists a tradition to the effect that the Aztecs invented the "atlatl" on the site referred to.. The eminent Robelo makes mention in his Dictionary of Aztec terms, of the surprising fact that by means of divers hieroglyphical elements is formed one orthological word...

We, taking all in consideration, believe in the hieroglyphic "atlatl" as being purely phonetic, because if it were representative it would necessarily have to preserve elements of the word "atlatl," the name of the place, which does not occur.

"CULHUACAN."-Place of the "colhua," the tribe. "Coltic" is twisted, and the hieroglyphic is a hill with its top bent over. The place is also referred to by the name of "Teoculhuacan" or "Tecolhuacan" in 

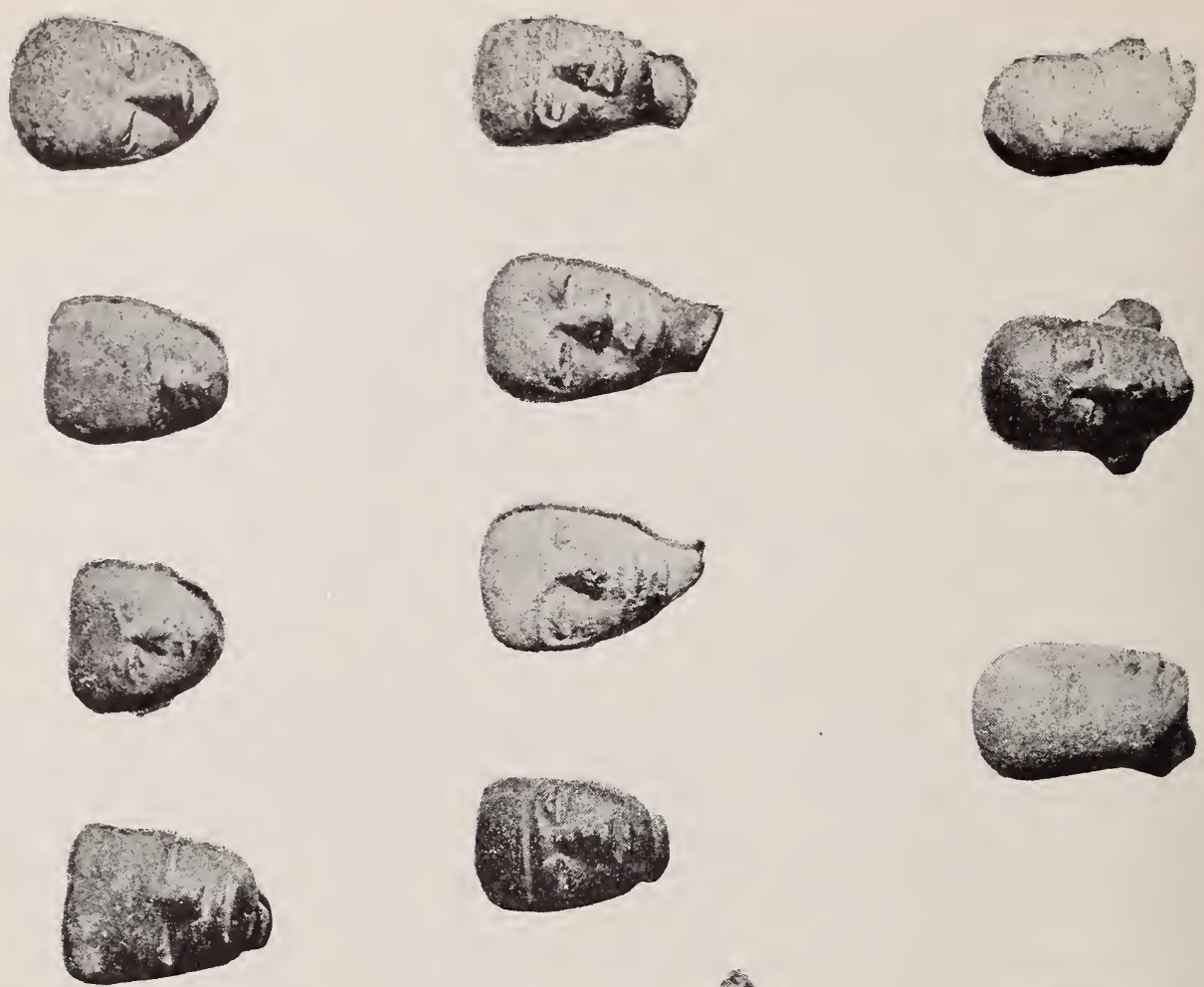

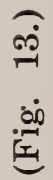
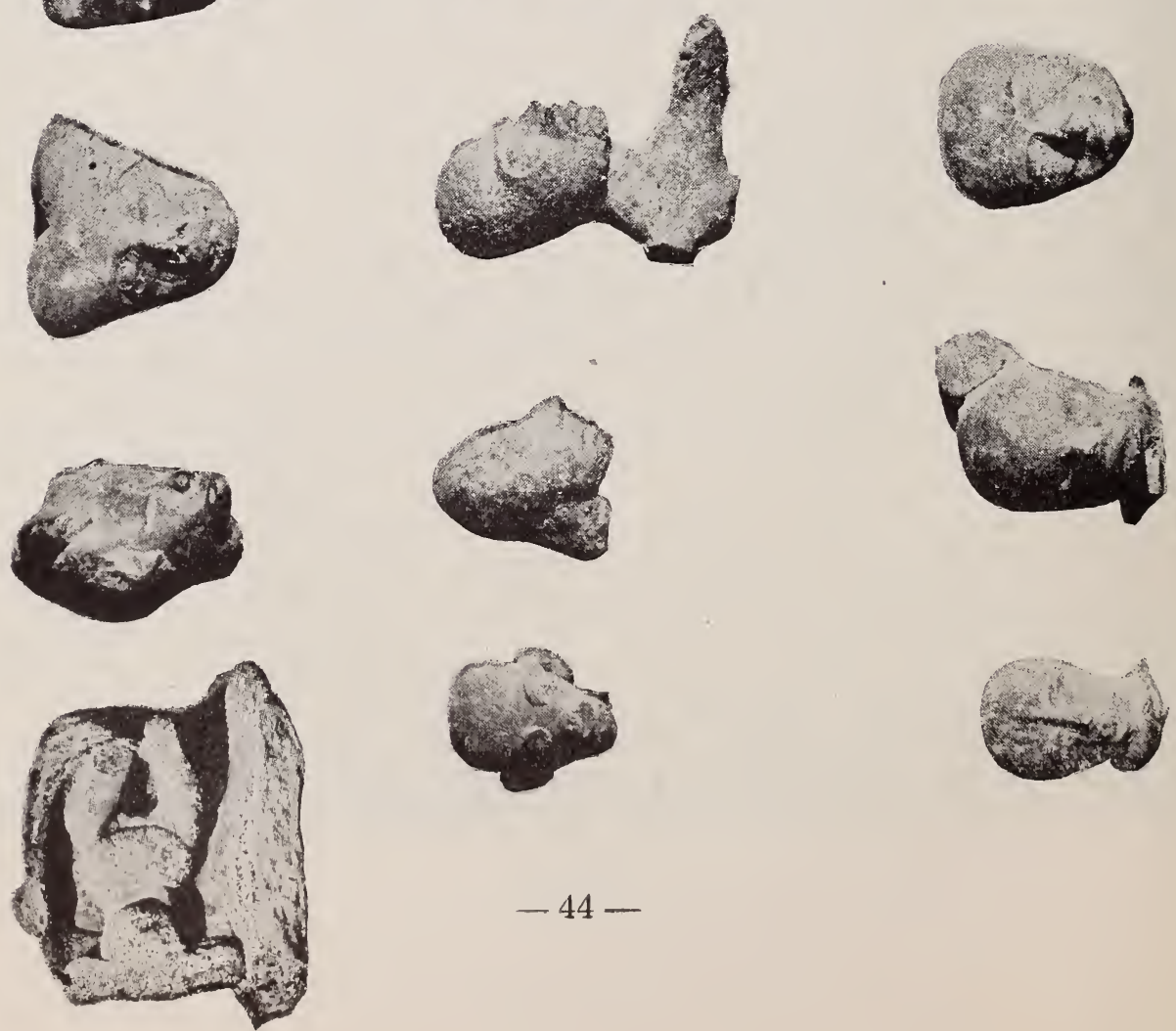
veneration of the god "Coltzin" who was adored and worshiped by the tribe.

"CACALCO."-(now San Joaquín) was a "tecpanecan" locality belonging to Tlacopam.-The place of crows: "cacalli" crow; "co" place.

"TEPALCATITLAN."-Place of pieces of earthenware (manufacture of earthen pots) or among the "tepacatl." "Titlan" maong. It is a fact that the "tepalcate" earthenware abounds here. "Cruztitlan." Is a word of hybrid nature. Place of the Cross.

"ARMASTITLAN."-Place or river of arms. A hybridism by which the native Indians of the present time designate the river of "Los Remedios."

"HUEYOTLI."-The old road.-CHuehuetl," old; "otil" road. At present there exists the remains of an old road now covered by surface soil, which has a course running to the eastward of Tepalcatitla, Ahuizoctla, and Amantla.

"XALPAN."-(San Pedro.) Place on the sand.-"Xalli," sand; "Icpan" on or over. This place is north of Tepalcatitla, and between the "Armastitla" river, and the ancient course, and therefore it is very sandy. We will now take up the subject of the Stratigraphy.

\section{STRATIGRAPHY.}

The study, description and classification of the stratums discovered during the works of exploration on the lands mentioned, are embodied in the representation as shown by Figure 2, and which accompanies same.

The stratigraphic process consisted of the following: Taking as a basis parcels of land containing 25, 50 and 100 square meters, these were divided into plots 15 meters long by 5 meters in width, as in this manner it is very easy to examine and make available for inspection the whole stratum and aluvion deposits by means of the shovel and the pick, bui when they are reached, only the geologist's pick and small adzes can be used for continuing the exploration work; furthermore, the earth which has been excavated is passed through a sand sifter immediately after being thrown out, by means of which and owing to the reduced dimensions of the perforation, no detail is lost.

The deposits in the stratum or stratums comprised within, are not regular but to the contrary, frequently discovered to exist in the form of subterranean pockets.

We have made excavations from the surface of the ground, down to a depth of seven meters,--but this only in very high places such as San Juanico (district of Popotla) and the maximum depth to which we have attained has been 5 meters 50 centimeters. Underlying the layers at this depth, appears the geological subsoil formations.

From the upper surface of the ground, or say from the vegetable earth down to 1 meter 95 centimeters, we find unmistakeably the specimens of a distinct Aztec type in the shap of deities, vases, objects of personal adornment, and rituals of the race.

At 1 meter 95 cts., varying to 2 meters 50 cts., we find the types of the "teotihuacans." 
At 2 meters $50 \mathrm{cts}$., varying to 4 meters $50 \mathrm{cts}$., we find the types of the "tecpanecans."

At 4 meters 50 cts., varying to 5 meters 50 cts., we find the types of the archaic or primitive dwellers in the valley.

In the Aztec stratums we have found the incenseburners ("amazalli popochcomitl); the remains of floorings constructed out of the porous tezontle stone and lime, and highly polished, as well as the wooden remants of roofiings, molding or cornices of well-baked clay, and also corners of stone.

In the "Teotihuacan" stratum, we have found the same colored floorings, as well as others constructed of the "adobe" mud-brick material. Also many black vases so characteristic of the "teotihuacan" period, together with other ones with a scraped decoration effect; bead necis ornaments, little jade necklaces, and the black ear-covering adjustments which were also characteristic of these ancient peoples.

In the "Tecpanec" stratums, we have found the following archaelogical specimens explained by this monograph:

Theoffering holders (huencahuatli ( commonly knwon as candlestichs have been found by us both in the Aztec and Teotihuacan stratums, with the same finish and style of modeling.

At the dividing line separating the "tecpanecan" stratum from the "teotihuacan" has been discovered a vase of unknown utility, showing on the border three chimneys not connected with the interior,--and with a portion of theedge turned downwards, as if it were for drinking purposes; always without coloring or burnishing, in the form of a truncated inverted cone, but well desiged; incomplete baking, and the human type on the part not occupied by the chimneys, is chub-checked with applications representing the head ornamentation, attached by the adhesive pastile method.

Immediately below the "tecpanecan" deposits, exist the archaic or primitive one, called "of the hills" by the International School of Archaeo$\log y$, although it is very improprely named, as we have stated on other occasions.

In conclusion, to have discovered and fixed the "Tecpanecan" horizon or stratum, has been a great triumph on the part of the archaeologist William Niven, and should be considered as a wonderful archaeological discovery of the greatest importance to antiquarian research.

México, D. F., December 1919.

R. MENA.

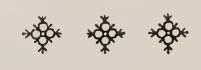






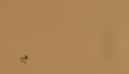

$\cdot$

ay $=$

$x$
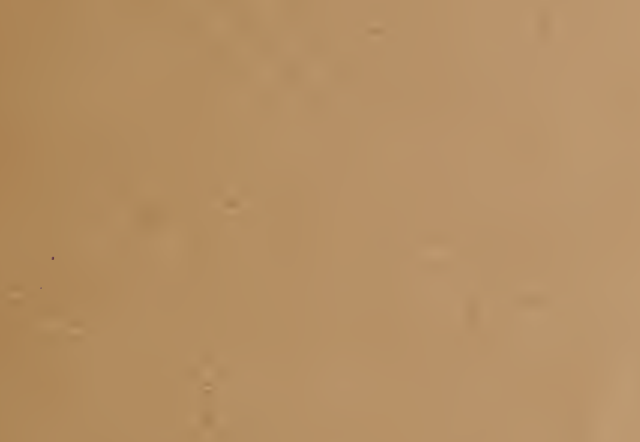

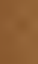

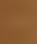

$+$
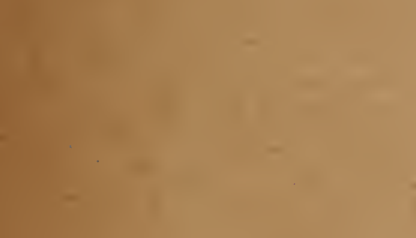

(n)

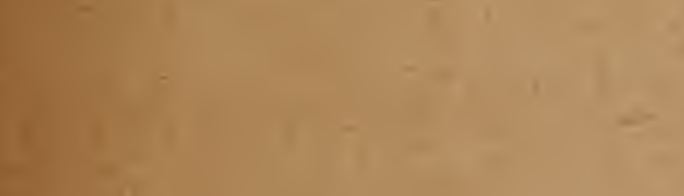

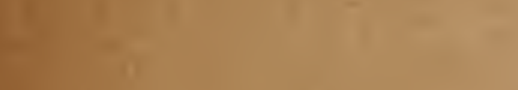
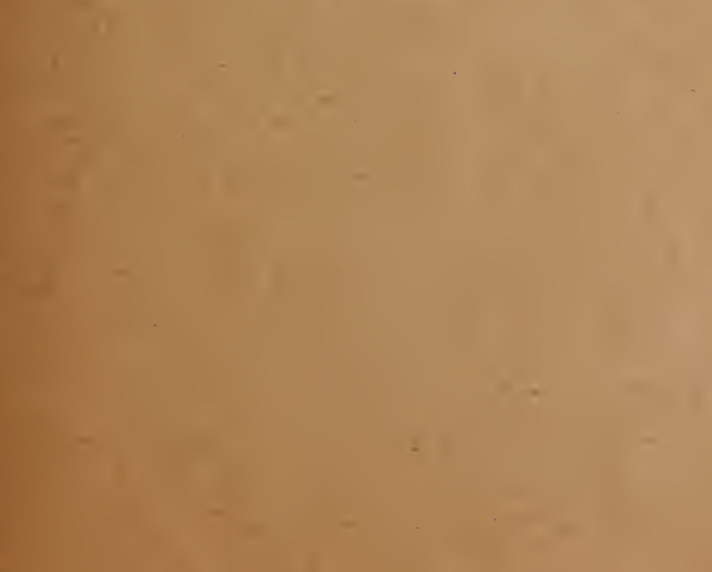

a.

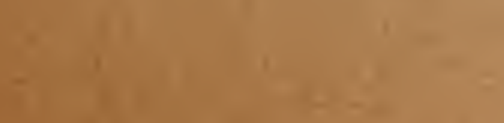

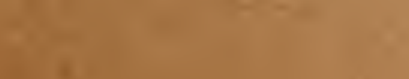





\section{MENA}

UN GRAN DESCUBRIMIENTO

ARQUEOLOGICO - LOS TECPANECA EN EL VALLE DE MEXICO 
SMITHSONIAN INSTITUTION LIBRARIES

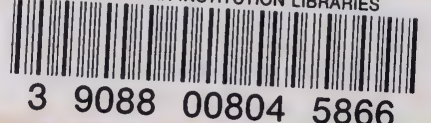

